\title{
A thermal immersed boundary-lattice Boltzmann method for moving-boundary flows with Dirichlet and Neumann conditions
}

\author{
Kosuke Suzuki $^{\mathrm{a}, *}$, Tsuyoshi Kawasaki ${ }^{\mathrm{b}}$, Naoki Furumachi ${ }^{\mathrm{b}, 1}$, Youming Tai ${ }^{\mathrm{b}, 2}$, \\ Masato Yoshino ${ }^{\mathrm{a}, \mathrm{c}}$ \\ ${ }^{a}$ Institute of Engineering, Academic Assembly, Shinshu University, Nagano 380-8553, \\ JAPAN \\ ${ }^{b}$ Department of Mechanical System Engineering, Faculty of Engineering, Shinshu \\ University, Nagano 380-8553, JAPAN \\ ${ }^{c}$ Institute of Carbon Science and Technology, Interdisciplinary Cluster for Cutting Edge \\ Research, Shinshu University, Nagano 380-8553, JAPAN
}

\begin{abstract}
We construct a simple immersed boundary-lattice Boltzmann method for movingboundary flows with heat transfer. On the basis of the immersed boundarylattice Boltzmann method for calculating the fluid velocity and the pressure fields presented in the previous work by Suzuki and Inamuro [Comput. Fluids, vol. 49, 2011, 173-187], the present method incorporates a lattice Boltzmann method for the temperature field combined with immersed boundary methods for satisfying thermal boundary conditions, i.e., the Dirichlet (iso-thermal) and Neumann (iso-heat-flux) conditions. We validate the present method through many benchmark problems including stationary and moving boundaries with iso-thermal and iso-heat-flux conditions, and we find that the present results have good agreement with other numerical results. Also, we investigate the internal heat effect through simulations of moving-boundary flows with heat transfer by using the present method. In addition, we apply the method to an interesting example of a moving-boundary flow with heat transfer, i.e., a two-
\end{abstract}

\footnotetext{
${ }^{*}$ Corresponding author

Email addresses: kosuzuki@shinshu-u.ac.jp (Kosuke Suzuki), masato@shinshu-u.ac.jp (Masato Yoshino)

${ }^{1}$ present affiliation: Central Japan Railway Company, Nagoya 450-6101, Japan

${ }^{2}$ present affiliation: Department of Aerospace Engineering, Graduate School of Engineering, Nagoya University, Nagoya 464-8603, Japan
} 
dimensional thermal flow in a heated channel with moving cold particles, which is a simplified model of ice slurry flow.

Keywords: Immersed boundary method, Lattice Boltzmann method, Moving-boundary flow, Heat transfer, Dirichlet condition, Neumann condition

\section{Introduction}

One of the important issues in computational fluid dynamics is to simulate moving-boundary flows efficiently and accurately. The simplest way is to approximate the boundary by staircase-like steps in a fixed Cartesian grid. In 5 applying the approximation to moving-boundary flows, however, it is required to construct new staircase-like steps in each time step, and the procedure is complicated and time-consuming in spite of its low accuracy. Other ways are body-fitted or unstructured-grid methods in which the grid conforms to the boundary. These methods can express arbitrary boundaries accurately and have traditionally been used for moving-boundary flows. However, due to re-meshing procedures, the algorithms of the methods are generally complicated, and also the computation costs are expensive. Recently, the immersed boundary method (IBM), which was proposed by Peskin [1,2] in 1970s in order to simulate blood flows in the heart, has been reconsidered as an efficient method for simulating moving-boundary flows on a fixed Cartesian grid. In the IBM, it is assumed that a fluid is filled in the inside of a boundary as well as in the outside of the boundary, and then appropriate body force is applied near the boundary in order to enforce the no-slip condition on the boundary. The IBM is a simple approach to moving-boundary flows, although certain techniques are necessary to determine the body force applied near the boundary. Various approaches and applications using the IBM were reviewed by Mittal and Iaccarino [3].

The idea of the IBM has been applied to moving-boundary flows with heat transfer. In the IBMs for heat transfer (referred to as thermal IBMs), an appropriate heat source/sink term is applied near the boundary in order to enforce the thermal boundary conditions, which are classified into two types, i.e., 
the Dirichlet (iso-thermal) condition and the Neumann (iso-heat-flux) condition. Several thermal IBMs for the two types of boundary conditions have been proposed. Kim and Choi [4] proposed a thermal IBM for both Dirichlet and Neumann conditions by introducing a heat source/sink term on the body surface or inside the body based on the finite volume approach on a staggered grid together with a fractional step method, and applied the thermal IBM to convection phenomena around stationary circular cylinders. Pacheco et al. [5] also proposed a thermal IBM for both Dirichlet and Neumann conditions based on the finite volume approach on a non-staggered grid, and validated it extensively through many heat-transfer problems with two-dimensional stationary boundaries which do not coincide with the grid. Pan [6] proposed a thermal IBM for the Dirichlet condition using volume-of-body function on multigrid Cartesian meshes, and validated it through force-convection and natural-convection problems around a stationary circular cylinder. Zhang et al. [7] presented a thermal IBM for both Dirichlet and Neumann conditions with a simple algorithm based on a direct-forcing approach, and applied it to heat-transfer problems with flows over not only a stationary cylinder but also an oscillating cylinder. Feng and Michaelides [8] developed a simple numerical method to solve the thermal interaction between particles and fluid in particulate flows. This method utilizes a thermal IBM for the Dirichlet condition. They validated it extensively through many heat-transfer problems with both stationary and moving boundaries, and applied it to the sedimentation of 56 heated circular particles. Wang et al. [9] proposed a thermal IBM (referred to as the multi-direct heat source scheme) in which the heat source/sink term is iteratively determined to enforce the Dirichlet condition on the boundary more accurately, and validated it through simulations of natural convection between concentric cylinders and of flow past a stationary circular cylinder. In addition, it was applied to flow past a staggered tube bank with heat transfer. Ren et al. [10] presented an efficient thermal IBM (referred to as the heat flux correction scheme) for the Neumann condition, in which the heat source/sink term is determined by the difference between the desired heat flux and the one calculated from the temporary temperature field without re- 
gard to the boundary. Numerical experiments for heat-transfer problems with stationary cylinders were conducted to validate the capability and efficiency of this method.

On the other hand, the lattice Boltzmann method (LBM) has been developed into an alternative and promising numerical scheme for simulating viscous fluid flows in the Cartesian grid without solving the Poisson equation for pressure fields [11]. Since both of the LBM and the IBM are based on the Cartesian grid, the LBM combined with the IBM (so-called IB-LBM) is well-suited to simulations of moving-boundary flows. Recently, several IB-LBMs which incorporate a thermal IBM (referred to as thermal IB-LBMs) have been proposed for solving heat-transfer problems with flows around complex geometries and/or moving boundaries. Jeong et al. [12] proposed a thermal IB-LBM using an equilibrium internal energy density approach to simulate natural convections in a 70 cavity with stationary circular and square cylinders. Kang and Hassan [13] combined the direct-forcing thermal IBM formulas with two types of LBMs, i.e., a hybrid model and a simplified double-population method, and validated them through two-dimensional convective heat-transfer problems with not only stationary but also moving boundaries. Zhang et al. [14] combined a thermal 75 IB-LBM with the discrete element method to simulate particulate flows with heat transfer. Eshghinejadfard and Thévenin [15] extended a thermal IB-LBM to three-dimensional particulate flows with heat transfer. Wu et al. [16] proposed a thermal IB-LBM in which the heat source/sink term at the next time step is taken as unknowns and iteratively corrected, and not only validated it through some two-dimensional heat-transfer problems but also applied it to a three-dimensional sedimentation of a single particle. While the above thermal IB-LBMs are for only the Dirichlet conditions, $\mathrm{Hu}$ et al. [17] proposed a thermal IB-LBM for Dirichlet, Neumann, and Robin (weighted combination of iso-thermal and iso-heat-flux) conditions, and tested it by some natural and ${ }_{85}$ forced convective problems including moving-boundary flows. Wang et al. [18] proposed a thermal IB-LBM for thermal flows with the Neumann conditions on the basis of the lattice Boltzmann flux solver, and applied it to several bench- 
marks of natural, forced, and mixed convection problems around a stationary circular cylinder.

As shown in the above-mentioned researches on the development of the thermal IB-LBM and its applications, there is growing concern about the thermal IB-LBM to solve heat-transfer problems with flows around complex geometries and/or moving boundaries efficiently. However, more work is needed to prove its effectiveness in simulations of moving-boundary flows with heat transfer. Especially, there is little to validate the thermal IB-LBM for iso-heat-flux movingboundary flows and to apply it to such problems.

The purposes of this study are to construct a simple thermal IB-LBM for solving heat-transfer problems with flows around moving boundaries efficiently, to validate it through many benchmark problems including stationary- and moving-boundary flows with the Dirichlet and Neumann conditions, and to apply it to an interesting example of a moving-boundary problem with heat transfer. In the present study, on the basis of the IB-LBM proposed by Suzuki and Inamuro [19] for calculating the fluid velocity and pressure fields, we construct a new thermal IB-LBM by combining a simple thermal LBM proposed by Inamuro et al. [20] and Yoshino and Inamuro [21] with two types of thermal IBMs, i.e., the multi-direct heat source scheme [9] and the heat flux correction scheme [10] for calculating the temperature field with the Dirichlet and Neumann conditions, respectively. It should be noted that the above two thermal IBMs have not been implemented in the framework of the LBM. The IB-LBM proposed by Suzuki and Inamuro [19] is a combination of the LBM and the multi-direct forcing scheme [22], which can enforce the no-slip condition accurately by determining the body force iteratively from the difference between the desired velocity and the flow velocity without regard to the boundary. The method has been extensively validated through many benchmark problems of moving-boundary flows in their work [19]. In addition, the method has been used for investigating the internal mass effect for the force and torque acting on the boundary, and it was revealed that the internal mass effect is very important in moving-boundary flows at high Reynolds numbers [19]. However, no one has 
investigated the importance of the internal mass effect for the rate of total heat tion of the problem in the framework of the IBM. In Section 3, we describe the present numerical method. In Section 4, we validate the present numerical method through thermal flows around a circular cylinder with the Dirichlet and Neumann conditions, Taylor-Couette flows with heat transfer, the sedicous fluid with heat transfer.

\subsection{Thermal fluid flow with a moving body represented by the IBM}

Let $\Omega_{\text {all }}$ be the entire domain of the system, $\Omega(t)$ be the closed domain inside the rigid body, and $\partial \Omega(t)$ be the surface of the body at time $t$ (see Fig. 1). We assume that an incompressible viscous fluid with the density $\rho_{\mathrm{f}}$, the viscosity $\mu$, the specific heat at constant pressure $c_{\mathrm{pf}}$, and the thermal conductivity $\lambda_{\mathrm{f}}$ is 
filled in the entire domain $\Omega_{\text {all }}$. We call the fluid inside $\Omega(t)$ the internal fluid and the fluid outside $\Omega(t)$ the external fluid. We assume that the no-slip condition on $\partial \Omega(t)$ is satisfied by body force $\boldsymbol{g}(\boldsymbol{x}, t)$ applied only on the neighborhood of $\partial \Omega(t)$ defined by $L_{\epsilon}(t)$ as shown in Fig. 1 . In addition, the thermal boundary conditions on $\partial \Omega(t)$ are satisfied by a heat source/sink term $q(\boldsymbol{x}, t)$ applied on $L_{\epsilon}(t)$, which represents the rate of heat transferred to the surrounding fluid per unit volume. The body force $\boldsymbol{g}$ and the heat source/sink term $q$ are determined by the IBM as explained in Section 3.

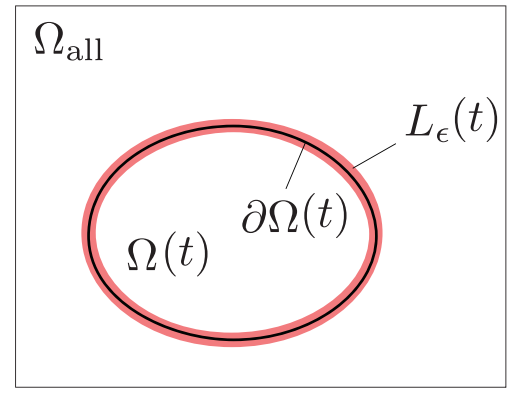

Figure 1: The system of a moving body in a fluid represented by the IBM.

In this study, we assume as follows:

- The density and the viscosity of the fluid are constant and uniform independently of the temperature.

- The viscous heat dissipation is negligibly small.

These assumptions have been adapted in most studies concerning the thermal IBM, and can be considered to be valid for the problems in this study. In addition, we consider only prescribed thermal boundary conditions which are determined independently of the thermal conduction inside the body. Therefore, the temperature field inside the body is completely imaginary, although we calculate it in both the inside and the outside of the body.

Under the above assumptions, the fluid flow is governed by the continuity 
equation and the Navier-Stokes equations for incompressible fluid as follows:

$$
\begin{aligned}
& \boldsymbol{\nabla} \cdot \boldsymbol{u}=0 \\
& \rho_{\mathrm{f}}\left[\frac{\partial \boldsymbol{u}}{\partial t}+(\boldsymbol{u} \cdot \boldsymbol{\nabla}) \boldsymbol{u}\right]=-\boldsymbol{\nabla} p+\mu \boldsymbol{\nabla}^{2} \boldsymbol{u}+\boldsymbol{g},
\end{aligned}
$$

where $\boldsymbol{u}(\boldsymbol{x}, t)$ and $p(\boldsymbol{x}, t)$ are the fluid velocity and the pressure at the point $\boldsymbol{x}$ at time $t$, respectively. It should be noted that $\boldsymbol{g}(\boldsymbol{x}, t)=\mathbf{0}$ if $\boldsymbol{x} \notin L_{\epsilon}(t)$. The temperature of the fluid is considered as a passive-scalar and governed by the following convection-diffusion equation:

$$
\rho_{\mathrm{f}} c_{\mathrm{pf}}\left[\frac{\partial T}{\partial t}+\boldsymbol{u} \cdot \boldsymbol{\nabla} T\right]=\lambda_{\mathrm{f}} \boldsymbol{\nabla}^{2} T+q,
$$

where $T(\boldsymbol{x}, t)$ is the temperature at the point $\boldsymbol{x}$ at time $t$. It should be noted that $q(\boldsymbol{x}, t)=0$ if $\boldsymbol{x} \notin L_{\epsilon}(t)$.

\subsection{Force and torque acting on the body and its motion}

The force $\boldsymbol{F}(t)$ applied by the external fluid to the body can be calculated by the negative of the sum of the body forces $\boldsymbol{g}(\boldsymbol{x}, t)$ and the internal mass effect [19] as below:

$$
\begin{gathered}
\boldsymbol{F}(t)=\boldsymbol{F}_{\text {tot }}(t)+\boldsymbol{F}_{\text {in }}(t), \\
\boldsymbol{F}_{\text {tot }}(t)=-\int_{\boldsymbol{x} \in L_{\epsilon}(t)} \boldsymbol{g}(\boldsymbol{x}, t) d \boldsymbol{x}, \\
\boldsymbol{F}_{\text {in }}(t)=\rho_{\mathrm{f}} \frac{d}{d t} \int_{\boldsymbol{x} \in \Omega(t)} \boldsymbol{u}(\boldsymbol{x}, t) d \boldsymbol{x} .
\end{gathered}
$$

Similarly, the torque $\boldsymbol{T}(t)$ acting on the body around a point $\boldsymbol{X}_{\mathrm{c}}(t)$ is calculated by

$$
\begin{gathered}
\boldsymbol{T}(t)=\boldsymbol{T}_{\mathrm{tot}}(t)+\boldsymbol{T}_{\mathrm{in}}(t), \\
\boldsymbol{T}_{\mathrm{tot}}(t)=-\int_{\boldsymbol{x} \in L_{\epsilon}(t)}\left[\boldsymbol{x}-\boldsymbol{X}_{\mathrm{c}}(t)\right] \times \boldsymbol{g}(\boldsymbol{x}, t) d \boldsymbol{x}, \\
\boldsymbol{T}_{\mathrm{in}}(t)=\rho_{\mathrm{f}} \frac{d}{d t} \int_{\boldsymbol{x} \in \Omega(t)}\left[\boldsymbol{x}-\boldsymbol{X}_{\mathrm{c}}(t)\right] \times \boldsymbol{u}(\boldsymbol{x}, t) d \boldsymbol{x} .
\end{gathered}
$$

In the case where the body freely moves, its motion is governed by the Newton-Euler equations with the force (4) and the torque (7) applied by the external fluid. The detailed description of the equations of the body motion is given by Suzuki and Inamuro [19]. 


\subsection{Rate of total heat transferred from the body to the surrounding fluid}

In a similar consideration to the force and torque acting on the body, the rate of total heat transferred from the body to the surrounding fluid can be calculated as bellow:

$$
\begin{gathered}
Q(t)=Q_{\mathrm{tot}}(t)+Q_{\mathrm{in}}(t), \\
Q_{\mathrm{tot}}(t)=\int_{\boldsymbol{x} \in L_{\epsilon}(t)} q(\boldsymbol{x}, t) d \boldsymbol{x}, \\
Q_{\mathrm{in}}(t)=-\rho_{\mathrm{f}} c_{\mathrm{pf}} \frac{d}{d t} \int_{\boldsymbol{x} \in \Omega(t)} T(\boldsymbol{x}, t) d \boldsymbol{x} .
\end{gathered}
$$

We call $Q_{\text {in }}$ the internal heat effect. It should be noted that the internal heat effect $Q_{\text {in }}$ is not zero when the temperature field is unsteady, e.g., movingboundary flows with heat transfer. The internal heat effect $Q_{\text {in }}$ in the simulation of moving-boundary flows with heat transfer by the thermal IBM is investigated in Section 4.3.

\section{Numerical method}

We use a thermal IB-LBM for solving Eqs. (1)-(3). The equations of the body motion are computed by the second-order Adams-Bashforth method. At each time step the force and the torque acting on the body are computed by Eqs. (4) and (7).

The present method is a combination of a simple thermal LBM with the multi-direct forcing scheme [22] to enforce the no-slip condition for the velocity and pressure fields, the multi-direct heat source scheme [9] to enforce the Dirichlet condition for the temperature field, and the heat flux correction scheme [10] to satisfy the Neumann condition for the temperature field. The no-slip condition for the velocity and pressure fields is enforced accurately by determining the body force iteratively from the difference between the desired velocity and the flow velocity without regard to the boundary. The Dirichlet condition for the temperature field is enforced accurately by determining the heat source/sink term iteratively in an analogous way to that for the no-slip condition. The 
Neumann condition for the temperature field is satisfied by determining the heat source/sink term from the difference between the desired heat flux and the one calculated from the temporary temperature field without regard to the boundary.

We use nondimensional variables defined in Appendix A. Note that the same notations as in Section 2 are used for the nondimensional variables. Although we only describe and implement two-dimensional examples in this paper, the present method can be extended to three-dimensions in a straightforward way.

\subsection{Thermal lattice Boltzmann method}

In the LBM, a modeled gas, which is composed of identical particles whose velocities are restricted to a finite set of vectors, is considered [11]. Twodimensional lattice with nine velocity vectors (D2Q9 model) is used in the present study. The D2Q9 model has the velocity vectors $\boldsymbol{c}_{i}=(0,0),(0, \pm 1)$, $( \pm 1,0),( \pm 1, \pm 1)$ for $i=1,2, \cdots, 9$. The evolution of the particle distribution functions $f_{i}(\boldsymbol{x}, t)$ and $g_{i}(\boldsymbol{x}, t)$ for the fluid motion and for the heat transfer, respectively, with the velocity $\boldsymbol{c}_{i}$ at the point $\boldsymbol{x}$ and time $t$ is computed by the following equations:

$$
\begin{aligned}
& f_{i}\left(\boldsymbol{x}+\boldsymbol{c}_{i} \Delta x, t+\Delta t\right)=f_{i}(\boldsymbol{x}, t)-\frac{1}{\tau_{f}}\left[f_{i}(\boldsymbol{x}, t)-f_{i}^{\mathrm{eq}}(\rho(\boldsymbol{x}, t), \boldsymbol{u}(\boldsymbol{x}, t))\right], \\
& g_{i}\left(\boldsymbol{x}+\boldsymbol{c}_{i} \Delta x, t+\Delta t\right)=g_{i}(\boldsymbol{x}, t)-\frac{1}{\tau_{g}}\left[g_{i}(\boldsymbol{x}, t)-g_{i}^{\mathrm{eq}}(T(\boldsymbol{x}, t), \boldsymbol{u}(\boldsymbol{x}, t))\right],
\end{aligned}
$$

where $\Delta x$ is a lattice spacing, $\Delta t$ is the time step during which the particles travel one lattice spacing, $f_{i}^{\text {eq }}$ and $g_{i}^{\text {eq }}$ are equilibrium distribution functions given below, and $\tau_{f}$ and $\tau_{g}$ are single relaxation times of $O(1)$. In Eqs. (13) and (14), $\boldsymbol{x}$ is a nondimensional position normalized by a characteristic length $\hat{H}_{0}, t$ is a nondimensional time normalized by a diffusive time scale $\hat{t}_{0}=\hat{H}_{0} / \hat{U}_{0}$ where

$\hat{U}_{0}$ is a characteristic flow speed, and $\boldsymbol{c}_{i}$ is a nondimensional particle velocity normalized by a characteristic particle speed $\hat{c}$. In the system where a rigid body moves in fluid, $\hat{H}_{0}$ is the scale of the body, and $\hat{U}_{0}$ is the speed of the body. In addition, we assume that $\hat{U}_{0} / \hat{c}$ is of $O(\Delta x)$. Note that $\Delta t=S h \Delta x$ where $S h=$ $\hat{H}_{0} /\left(\hat{t}_{0} \hat{c}\right)=\hat{U}_{0} / \hat{c}=O(\Delta x)$ (see Appendix A). The equilibrium distribution 
functions $f_{i}^{\text {eq }}$ for the fluid motion [24] and $g_{i}^{\text {eq }}$ for the heat transfer $[20,21]$ are given by

$$
\begin{aligned}
f_{i}^{\mathrm{eq}}(\rho, \boldsymbol{u}) & =E_{i} \rho\left[1+3 \boldsymbol{c}_{i} \cdot \boldsymbol{u}+\frac{9}{2}\left(\boldsymbol{c}_{i} \cdot \boldsymbol{u}\right)^{2}-\frac{3}{2} \boldsymbol{u} \cdot \boldsymbol{u}\right], \\
g_{i}^{\mathrm{eq}}(T, \boldsymbol{u}) & =E_{i} T\left[1+3 \boldsymbol{c}_{i} \cdot \boldsymbol{u}\right]
\end{aligned}
$$

where $E_{1}=4 / 9, E_{2}=\cdots=E_{5}=1 / 9$, and $E_{6}=\cdots=E_{9}=1 / 36$ for the D2Q9 model. The above equilibrium distribution function $g_{i}^{\mathrm{eq}}$ is the simplest one which can recover the convection-diffusion equation (3). The density $\rho(\boldsymbol{x}, t)$, the pressure $p(\boldsymbol{x}, t)$, the fluid velocity $\boldsymbol{u}(\boldsymbol{x}, t)$, and the temperature $T(\boldsymbol{u}, t)$ are calculated by

$$
\begin{gathered}
\rho=\sum_{i=1}^{9} f_{i}, \\
p=\frac{\rho}{3}, \\
\boldsymbol{u}=\frac{1}{\rho} \sum_{i=1}^{9} f_{i} \boldsymbol{c}_{i}, \\
T=\sum_{i=1}^{9} g_{i} .
\end{gathered}
$$

In addition, the heat-flux vector, i.e., the temperature gradient can be calculated as follows [21]:

$$
\boldsymbol{h}=-\lambda_{\mathrm{f}} \boldsymbol{\nabla} T=\sum_{i=1}^{9} g_{i}\left(\boldsymbol{c}_{i}-\boldsymbol{u}\right),
$$

where the thermal conductivity $\lambda_{\mathrm{f}}$ is given by

$$
\lambda_{\mathrm{f}}=\frac{1}{3} \tau_{g} \Delta x
$$

The kinematic viscosity $\nu$ and the thermal diffusivity $\alpha$ are given by

$$
\begin{aligned}
\nu & =\frac{1}{3}\left(\tau_{f}-\frac{1}{2}\right) \Delta x, \\
\alpha & =\frac{1}{3}\left(\tau_{g}-\frac{1}{2}\right) \Delta x .
\end{aligned}
$$


When external body force $\boldsymbol{g}(\boldsymbol{x}, t)$ and an external heat source/sink term $q(\boldsymbol{x}, t)$ are applied, the evolution equations (13) and (14) of the particle distribution functions $f_{i}(\boldsymbol{x}, t)$ and $g_{i}(\boldsymbol{x}, t)$ can be calculated in a stepwise fashion as follows:

1. $f_{i}(\boldsymbol{x}, t)$ and $g_{i}(\boldsymbol{x}, t)$ are evolved without the body force and the heat source/sink term as follows:

$$
\begin{aligned}
& f_{i}^{*}\left(\boldsymbol{x}+\boldsymbol{c}_{i} \Delta x, t+\Delta t\right)=f_{i}(\boldsymbol{x}, t)-\frac{1}{\tau_{f}}\left[f_{i}(\boldsymbol{x}, t)-f_{i}^{\mathrm{eq}}(\rho(\boldsymbol{x}, t), \boldsymbol{u}(\boldsymbol{x}, t))\right], \\
& g_{i}^{*}\left(\boldsymbol{x}+\boldsymbol{c}_{i} \Delta x, t+\Delta t\right)=g_{i}(\boldsymbol{x}, t)-\frac{1}{\tau_{g}}\left[g_{i}(\boldsymbol{x}, t)-g_{i}^{\mathrm{eq}}(T(\boldsymbol{x}, t), \boldsymbol{u}(\boldsymbol{x}, t))\right] .
\end{aligned}
$$

2. $f_{i}^{*}$ and $g_{i}^{*}$ are corrected by the body force and the heat source/sink term as follows:

$$
\begin{aligned}
& f_{i}(\boldsymbol{x}, t+\Delta t)=f_{i}^{*}(\boldsymbol{x}, t+\Delta t)+3 \Delta x E_{i} \rho^{*}(\boldsymbol{x}, t+\Delta t) \boldsymbol{c}_{i} \cdot \boldsymbol{g}(\boldsymbol{x}, t+\Delta t) \\
& g_{i}(\boldsymbol{x}, t+\Delta t)=g_{i}^{*}(\boldsymbol{x}, t+\Delta t)+\frac{\Delta x}{\rho_{\mathrm{f}} c_{\mathrm{pf}}} E_{i} q(\boldsymbol{x}, t+\Delta t)
\end{aligned}
$$

where $\rho^{*}(\boldsymbol{x}, t+\Delta t)$ is the temporary density calculated from Eq. (17) by using $f_{i}^{*}$. Since in the thermal LBM $\alpha$ and $\lambda_{\mathrm{f}}$ are given by Eqs. (24) and (22), respectively, the value of $\rho_{\mathrm{f}} c_{\mathrm{pf}}$ in the right-hand side of Eq. (28) can be calculated as $\rho_{\mathrm{f}} c_{\mathrm{pf}}=\lambda_{\mathrm{f}} / \alpha$.

As described in Ref. [25], it is found that the asymptotic expansions of $p$, $\boldsymbol{u}$, and $T$ with respect to $\Delta x$ can be expressed by $p=\rho_{\mathrm{f}} / 3+(\Delta x)^{2} p^{(2)}+$ $(\Delta x)^{3} p^{(3)}+(\Delta x)^{4} p^{(4)}+\cdots, \boldsymbol{u}=(\Delta x) \boldsymbol{u}^{(1)}+(\Delta x)^{2} \boldsymbol{u}^{(2)}+(\Delta x)^{3} \boldsymbol{u}^{(3)}+\cdots$, and $T=T^{(0)}+(\Delta x) T^{(1)}+(\Delta x)^{2} T^{(2)}+\cdots$, and $p^{(2)}, \boldsymbol{u}^{(1)}$, and $T^{(0)}$ satisfy the continuity equation (1), the Navier-Stokes equations (2), and the convectiondiffusion equation (3) for incompressible viscous fluid, while $p^{(3)}, \boldsymbol{u}^{(2)}$, and $T^{(1)}$ are zero with appropriate initial and boundary conditions [26]. Therefore, the solutions of Eqs. (25)-(28) and (15)-(20) give the pressure, the fluid velocity, 


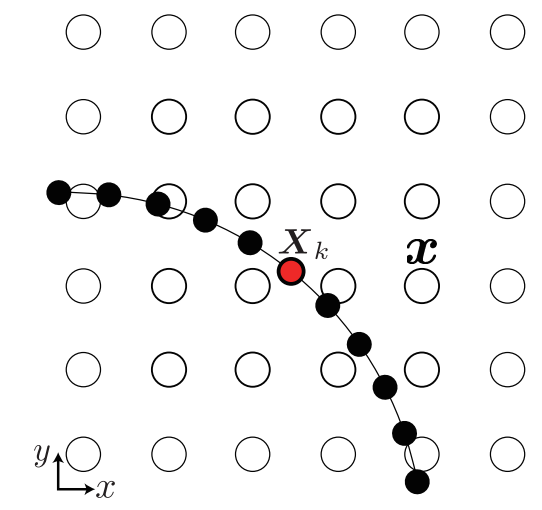

Figure 2: Illustration of an arrangement of boundary points $\boldsymbol{X}_{k}$ and lattice points $\boldsymbol{x}$.

and the temperature for incompressible viscous fluid flows and heat transfer with relative errors of $O\left[(\Delta x)^{2}\right]$ (see Refs. [20, 27]).

\subsection{Immersed boundary method for fluid motion}

In the IBM for fluid motion, the body force is applied on lattice points near the boundary in order to enforce the no-slip condition on the boundary. In this paper, we use the multi-direct forcing scheme proposed by Wang et al. [22]. Although the IBM for fluid motion is the same method as presented in the previous work [19], we describe it again for preparing some formulations used in the thermal IBM explained bellow and for clearly describing the whole algorithm for fluid motion and heat transfer together.

Supposing that $f_{i}(\boldsymbol{x}, t), \rho(\boldsymbol{x}, t)$, and $\boldsymbol{u}(\boldsymbol{x}, t)$ are known, the temporary $f_{i}^{*}(\boldsymbol{x}, t+$ $\Delta t), \rho^{*}(\boldsymbol{x}, t+\Delta t)$, and $\boldsymbol{u}_{i}^{*}(\boldsymbol{x}, t+\Delta t)$ can be calculated by Eqs. (25), (17), and (19), respectively. Let $\boldsymbol{X}_{k}(t+\Delta t)$ and $\boldsymbol{U}_{k}(t+\Delta t)(k=1,2, \cdots, N)$ be the position of the Lagrangian points of the moving boundary and the boundary velocity at the points, respectively. Note that the moving boundary is represented by $N$ points, and the boundary Lagrangian points $\boldsymbol{X}_{k}$ generally differ from the background lattice points $\boldsymbol{x}$ (see Fig. 2). Then, the temporary velocities $\boldsymbol{u}^{*}\left(\boldsymbol{X}_{k}, t+\Delta t\right)$ at the boundary Lagrangian points $\boldsymbol{X}_{k}$ are interpolated by 


$$
\boldsymbol{u}^{*}\left(\boldsymbol{X}_{k}, t+\Delta t\right)=\sum_{\boldsymbol{x}} \boldsymbol{u}^{*}(\boldsymbol{x}, t+\Delta t) W\left(\boldsymbol{x}-\boldsymbol{X}_{k}\right)(\Delta x)^{2},
$$

where $\sum_{\boldsymbol{x}}$ describes the summation over all lattice points $\boldsymbol{x}$, and $W$ is a weighting function proposed by Peskin [28] given by

$$
\begin{gathered}
W(x, y)=\frac{1}{\Delta x} w\left(\frac{x}{\Delta x}\right) \cdot \frac{1}{\Delta x} w\left(\frac{y}{\Delta x}\right), \\
w(r)= \begin{cases}\frac{1}{8}\left(3-2|r|+\sqrt{1+4|r|-4 r^{2}}\right), & |r| \leq 1, \\
\frac{1}{8}\left(5-2|r|-\sqrt{-7+12|r|-4 r^{2}}\right), & 1 \leq|r| \leq 2, \\
0, & \text { otherwise. }\end{cases}
\end{gathered}
$$

The body force $\boldsymbol{g}(\boldsymbol{x}, t+\Delta t)$ is determined by the following iterative procedure.

Step 0. Compute the initial value of the body force at the boundary Lagrangian points by

$$
\boldsymbol{g}_{0}\left(\boldsymbol{X}_{k}, t+\Delta t\right)=\rho_{\mathrm{f}} S h \frac{\boldsymbol{U}_{k}-\boldsymbol{u}^{*}\left(\boldsymbol{X}_{k}, t+\Delta t\right)}{\Delta t} .
$$

Step 1. Compute the body force at the lattice points of the $\ell$ th iteration by

$$
\boldsymbol{g}_{\ell}(\boldsymbol{x}, t+\Delta t)=\sum_{k=1}^{N} \boldsymbol{g}_{\ell}\left(\boldsymbol{X}_{k}, t+\Delta t\right) W\left(\boldsymbol{x}-\boldsymbol{X}_{k}\right) \Delta V,
$$

where the body force is not added to one boundary Lagrangian point but a small volume element whose volume is described as $\Delta V$. In this method, $\Delta V$ is taken as $S / N \times \Delta x$ where $S$ is the total length of the boundary, and $S / N$ is taken to be approximately equal to $\Delta x$. It should be noted that while Peskin [28] imposed $S / N<0.5(\Delta x)$ in order to avoid leaks, we found in our preliminary calculations that results with $S / N$ in the range of $0.5(\Delta x)$ to $1.0(\Delta x)$ almost coincide with each other.

Step 2. Correct the velocity at the lattice points by

$$
\boldsymbol{u}_{\ell}(\boldsymbol{x}, t+\Delta t)=\boldsymbol{u}^{*}(\boldsymbol{x}, t+\Delta t)+\frac{1}{\rho_{\mathrm{f}}} \frac{\Delta t}{S h} \boldsymbol{g}_{\ell}(\boldsymbol{x}, t+\Delta t) .
$$


Step 3. Interpolate the velocity at the boundary Lagrangian points with

$$
\boldsymbol{u}_{\ell}\left(\boldsymbol{X}_{k}, t+\Delta t\right)=\sum_{\boldsymbol{x}} \boldsymbol{u}_{\ell}(\boldsymbol{x}, t+\Delta t) W\left(\boldsymbol{x}-\boldsymbol{X}_{k}\right)(\Delta x)^{2} .
$$

Step 4. Correct the body force with

$$
\boldsymbol{g}_{\ell+1}\left(\boldsymbol{X}_{k}, t+\Delta t\right)=\boldsymbol{g}_{\ell}\left(\boldsymbol{X}_{k}, t+\Delta t\right)+\rho_{\mathrm{f}} S h \frac{\boldsymbol{U}_{k}-\boldsymbol{u}_{\ell}\left(\boldsymbol{X}_{k}, t+\Delta t\right)}{\Delta t},
$$

and go to Step 1.

In the previous work [19], it was reported that $\boldsymbol{g}_{\ell=5}(\boldsymbol{x}, t+\Delta t)$ is enough to keep no-slip condition on the boundary. Therefore, we iterate the above procedure until $\ell=5$ in the following computations.

\subsection{Thermal immersed boundary method}

In the thermal IBM, the heat source/sink term is applied on lattice points near the boundary in order to enforce the thermal boundary conditions on the boundary, i.e., the Dirichlet and Neumann conditions. In this paper, we use the multi-direct heat source scheme proposed by Wang et al. [9] for the Dirichlet condition, and the heat flux correction scheme proposed by Ren et al. [10] for the Neumann condition.

\subsubsection{Multi-direct heat source scheme}

This scheme is based on the same concept as the multi-direct forcing method, that is, the heat source/sink term is calculated iteratively to enforce the Dirichlet condition strongly. Supposing that $g_{i}(\boldsymbol{x}, t), T(\boldsymbol{x}, t)$, and $\boldsymbol{u}(\boldsymbol{x}, t)$ are known, the temporary $g_{i}^{*}(\boldsymbol{x}, t+\Delta t)$ and $T^{*}(\boldsymbol{x}, t+\Delta t)$ can be calculated by Eqs. (26) and (20), respectively. Then, the temporary temperatures $T^{*}\left(\boldsymbol{X}_{k}, t+\Delta t\right)$ at the boundary Lagrangian points $\boldsymbol{X}_{k}$ are interpolated in the same way as Eq. (29), i.e.,

$$
T^{*}\left(\boldsymbol{X}_{k}, t+\Delta t\right)=\sum_{\boldsymbol{x}} T^{*}(\boldsymbol{x}, t+\Delta t) W\left(\boldsymbol{x}-\boldsymbol{X}_{k}\right)(\Delta x)^{2} .
$$

Let $T_{k}^{\mathrm{d}}(t+\Delta t)$ be the desired temperatures at the Lagrangian points $\boldsymbol{X}_{k}$ given as the Dirichlet condition. The heat source/sink term $q(\boldsymbol{x}, t+\Delta t)$ is determined by the following iterative procedure. 
Step 0. Compute the initial value of the heat source/sink term at the boundary Lagrangian points by

$$
q_{0}\left(\boldsymbol{X}_{k}, t+\Delta t\right)=\rho_{\mathrm{f}} c_{\mathrm{pf}} S h \frac{T_{k}^{\mathrm{d}}-T^{*}\left(\boldsymbol{X}_{k}, t+\Delta t\right)}{\Delta t} .
$$

Step 1. Compute the heat source/sink term at the lattice points of the $m$ th iteration by

$$
q_{m}(\boldsymbol{x}, t+\Delta t)=\sum_{k=1}^{N} q_{m}\left(\boldsymbol{X}_{k}, t+\Delta t\right) W\left(\boldsymbol{x}-\boldsymbol{X}_{k}\right) \Delta V .
$$

Step 2. Correct the temperature at the lattice points by

$$
T_{m}(\boldsymbol{x}, t+\Delta t)=T^{*}(\boldsymbol{x}, t+\Delta t)+\frac{1}{\rho_{\mathrm{f}} c_{\mathrm{pf}}} \frac{\Delta t}{S h} q_{m}(\boldsymbol{x}, t+\Delta t) .
$$

Step 3. Interpolate the temperature at the boundary Lagrangian points with

$$
T_{m}\left(\boldsymbol{X}_{k}, t+\Delta t\right)=\sum_{\boldsymbol{x}} T_{m}(\boldsymbol{x}, t+\Delta t) W\left(\boldsymbol{x}-\boldsymbol{X}_{k}\right)(\Delta x)^{2} .
$$

Step 4. Correct the heat source/sink term with

$$
q_{m+1}\left(\boldsymbol{X}_{k}, t+\Delta t\right)=q_{m}\left(\boldsymbol{X}_{k}, t+\Delta t\right)+\rho_{\mathrm{f}} c_{\mathrm{pf}} S h \frac{T_{k}^{\mathrm{d}}-T_{m}\left(\boldsymbol{X}_{k}, t+\Delta t\right)}{\Delta t},
$$

and go to Step 1.

From preliminary computations, we found that $q_{m=5}(\boldsymbol{x}, t+\Delta t)$ is enough to keep

the Dirichlet condition on the boundary points (see Table 1 in Section 4.1.1). Therefore, we iterate the above procedure until $m=5$ in the following computations.

\subsubsection{Heat flux correction method}

Supposing that $g_{i}(\boldsymbol{x}, t), T(\boldsymbol{x}, t)$, and $\boldsymbol{u}(\boldsymbol{x}, t)$ are known, the temporary $g_{i}^{*}(\boldsymbol{x}, t+$ $\Delta t)$ can be calculated by Eq. (26). In addition, the temporary heat-flux vector $\boldsymbol{h}^{*}(\boldsymbol{x}, t+\Delta t)$ is calculated by Eq. (21) as follows:

$$
\boldsymbol{h}^{*}(\boldsymbol{x}, t+\Delta t)=\sum_{i=1}^{9} g_{i}^{*}(\boldsymbol{x}, t+\Delta t)\left[\boldsymbol{c}_{i}-\boldsymbol{u}(\boldsymbol{x}, t+\Delta t)\right] .
$$


The temporary heat-flux vectors $\boldsymbol{h}^{*}\left(\boldsymbol{X}_{k}, t+\Delta t\right)$ at the boundary Lagrangian points $\boldsymbol{X}_{k}$ are interpolated in the same way as Eq. (29), i.e.,

$$
\boldsymbol{h}^{*}\left(\boldsymbol{X}_{k}, t+\Delta t\right)=\sum_{\boldsymbol{x}} \boldsymbol{h}^{*}(\boldsymbol{x}, t+\Delta t) W\left(\boldsymbol{x}-\boldsymbol{X}_{k}\right)(\Delta x)^{2} .
$$

Let $\boldsymbol{n}_{k}(t+\Delta t)$ be the unit normal vectors of the boundary at the Lagrangian points $\boldsymbol{X}_{k}(t+\Delta t)$ pointing to the external fluid. Then, the temporary heat-flux in the normal direction is given by

$$
h_{\mathrm{n}}^{*}\left(\boldsymbol{X}_{k}, t+\Delta t\right)=\boldsymbol{n}_{k} \cdot \boldsymbol{h}^{*}\left(\boldsymbol{X}_{k}, t+\Delta t\right) .
$$

It should be noted that in the original heat flux correction method proposed by Ren et al. [10], the temporary heat-flux is calculated by the second-order central difference approximation for the temperature, while the present method uses Eq. (43). Both ways should have the same accuracy, but lattice points used for calculating the temporary heat-flux are different. The number of the points used in the present way is larger than that used in the way of Ren et 265 al. [10], and therefore it is expected that the present way is more stable. In our preliminary calculations, however, both ways gave almost the same results and did not show any instability. Therefore, either way might be fine in twodimensional calculations.

Let $H_{k}^{\mathrm{d}}(t+\Delta t)$ be the desired heat fluxes at the Lagrangian points $\boldsymbol{X}_{k}$ in its normal direction given as the Neumann condition. The heat source/sink term $q(\boldsymbol{x}, t+\Delta t)$ is determined by

$$
q\left(\boldsymbol{X}_{k}, t+\Delta t\right)=2 \frac{H_{k}^{\mathrm{d}}(t+\Delta t)-h_{\mathrm{n}}^{*}\left(\boldsymbol{X}_{k}, t+\Delta t\right)}{\Delta x},
$$

where the coefficient 2 in the right-hand side of the above equation means that the heat flux due to the difference of $H_{k}(t+\Delta t)$ and $h_{\mathrm{n}}^{*}\left(\boldsymbol{X}_{k}, t+\Delta t\right)$ should affect the internal fluid as much as the external fluid. Then, the heat source/sink terms at the Lagrangian points are distributed to the lattice points in the same way as Eq. (39) as follows:

$$
q(\boldsymbol{x}, t+\Delta t)=\sum_{k=1}^{N} q\left(\boldsymbol{X}_{k}, t+\Delta t\right) W\left(\boldsymbol{x}-\boldsymbol{X}_{k}\right) \Delta V .
$$




\subsection{Algorithm of computation}

270

the algorithm of computation by the present numerical method is summarized as below.

0 . Suppose the initial value of $f_{i}(\boldsymbol{x}, 0)$ and $g_{i}(\boldsymbol{x}, 0)$, and compute $\rho(\boldsymbol{x}, 0)$, $\boldsymbol{u}(\boldsymbol{x}, 0)$, and $T(\boldsymbol{x}, 0)$ by Eqs. (17), (19), and (20), respectively.

275

1. Set $\boldsymbol{X}_{k}(t+\Delta t), \boldsymbol{n}_{k}(t+\Delta t)$, and $\boldsymbol{U}_{k}(t+\Delta t)$ in a predetermined manner.

2. Compute $f_{i}^{*}(\boldsymbol{x}, t+\Delta t)$ by Eq. (25), and $\rho^{*}(\boldsymbol{x}, t+\Delta t)$ and $\boldsymbol{u}^{*}(\boldsymbol{x}, t+\Delta t)$ by Eqs. (17) and (19), respectively. Then, compute $\boldsymbol{u}^{*}\left(\boldsymbol{X}_{k}, t+\Delta t\right)$ by Eq. (29).

3. Compute $\boldsymbol{g}(\boldsymbol{x}, t+\Delta t)$ by Eqs. (32)-(36).

4. Compute $f_{i}(\boldsymbol{x}, t+\Delta t)$ by Eq. (27), and $\rho(\boldsymbol{x}, t+\Delta t)$ and $\boldsymbol{u}(\boldsymbol{x}, t+\Delta t)$ by Eqs. (17) and (19), respectively.

If the Dirichlet condition is considered for the heat transfer,

5. Set $T_{k}^{\mathrm{d}}(t+\Delta t)$.

6. Compute $g_{i}^{*}(\boldsymbol{x}, t+\Delta t)$ by Eq. (26) and $T^{*}(\boldsymbol{x}, t+\Delta t)$ by Eq. (20). Then, compute $T^{*}\left(\boldsymbol{X}_{k}, t+\Delta t\right)$ by Eq. (37).

7. Compute $q(\boldsymbol{x}, t+\Delta t)$ by Eqs. (38)-(42).

8. Compute $g_{i}(\boldsymbol{x}, t+\Delta t)$ by Eq. (28) and $T(\boldsymbol{x}, t+\Delta t)$ by Eq. (20).

9. Advance one time step and return to 1 .

If the Neumann condition is considered for the heat transfer,

5. Set $H_{k}^{\mathrm{d}}(t+\Delta t)$.

6. Compute $g_{i}^{*}(\boldsymbol{x}, t+\Delta t)$ by Eq. (26) and $\boldsymbol{h}^{*}(\boldsymbol{x}, t+\Delta t)$ by Eq. (21). Then, compute $\boldsymbol{h}^{*}\left(\boldsymbol{X}_{k}, t+\Delta t\right)$ by Eq. (44).

7. Compute $h_{\mathrm{n}}\left(\boldsymbol{X}_{k}, t+\Delta t\right)$ by Eq. (45).

8. Compute $q(\boldsymbol{x}, t+\Delta t)$ by Eqs. (46) and (47).

9. Compute $g_{i}(\boldsymbol{x}, t+\Delta t)$ by Eq. (28) and $T(\boldsymbol{x}, t+\Delta t)$ by Eq. (20).

10. Advance one time step and return to 1 . 
It should be noted that when the body is moved by the force and the torque acting on the body, we calculate the force and the torque, compute the equations of the body motion, and then determine $\boldsymbol{X}_{k}(t+\Delta t), \boldsymbol{n}_{k}(t+\Delta t)$, and $\boldsymbol{U}_{k}(t+\Delta t)$ instead of the implementation 1 in the above algorithm.

\section{Numerical validations}

In this section, we examine the accuracy of the present thermal IB-LBM by applying it to some benchmark problems for the Dirichlet and Neumann conditions. In addition, we investigate the internal heat effect through simulations of moving-boundary flows with heat transfer by using the present thermal IB-LBM.

\subsection{For the Dirichlet condition}

\subsubsection{Thermal flow around an iso-thermal circular cylinder}

We consider a thermal flow around an iso-thermal circular cylinder. The diameter of the circular cylinder is $D_{\mathrm{s}}$. The computational domain is $\left[-10 D_{\mathrm{s}}, 18 D_{\mathrm{s}}\right] \times$ $\left[-10 D_{\mathrm{s}}, 10 D_{\mathrm{s}}\right]$. The center of the circular cylinder is located at $(x, y)=(0,0)$. In the inlet $\left(x=-10 D_{\mathrm{s}}\right)$, a uniform iso-thermal flow in the $x$-direction with a speed $U_{\infty}$ and at a temperature $T_{\infty}=0$ is imposed. In the outlet $\left(x=18 D_{\mathrm{s}}\right)$, the non-reflecting condition [29] is imposed, i.e., $\partial \phi / \partial t+C \partial \phi / \partial x=0$ for $\phi=\boldsymbol{u}$ and $T$, where $C=\Delta x / \Delta t$. In the bottom $\left(y=-10 D_{\mathrm{s}}\right)$ and top $\left(y=10 D_{\mathrm{s}}\right)$ boundaries, the slip condition $(\partial u / \partial y=0$ and $v=0$ where $\boldsymbol{u}=(u, v))$ and the adiabatic condition $(\partial T / \partial y=0)$ are applied. The circular cylinder is stationary and iso-thermal at a temperature $T_{\mathrm{s}}=1$. The governing parameters of this system are the Reynolds number defined by $R e=U_{\infty} D_{\mathrm{s}} / \nu$ and the Prandtl number defined by $\operatorname{Pr}=\nu / \alpha$. We set $\operatorname{Re}=20$ and $\operatorname{Pr}=0.73$ in order to compare the present results with other numerical results $[9,30-32]$. We consider the steady state of this system.

In the lattice Boltzmann simulation, the boundary conditions have to be implemented in terms of the particle distribution functions. In the inlet, the 

Inamuro [21] are used for imposing the flow speed $U_{\infty}$ and the temperature $T_{\infty}$, respectively. In the outlet, the velocity and the temperature are determined as $\phi\left(18 D_{\mathrm{s}}, y, t+\Delta t\right)=\phi\left(18 D_{\mathrm{s}}-\Delta x, y, t\right)$ for $\phi=\boldsymbol{u}$ and $T$ by using the first-order upwind difference scheme, and we impose them in the same way as the inlet. In the bottom and top boundaries, the mirror reflection of $f_{i}$ and the iso-flux condition proposed by Yoshino and Inamuro [21] are used for imposing the slip condition and the adiabatic condition, respectively. The boundary conditions on the surface of the circular cylinder are implemented by the present thermal IB-LBM. The steady state is determined when four significant digits for the mean Nusselt number $\overline{N u}$ (shown below) converge to constant values.

At first, we examine the effect of the number of iterations $m$ in the multidirect heat source scheme. In this simulation, we set $D_{\mathrm{s}}=50 \Delta x$, which is comparable with that in Refs. [31, 32], and $N=204$. In addition, we set $U_{\infty}=0.04, \tau_{f}=0.8000$, and $\tau_{g}=0.9110$. We define the maximum and mean errors from the iso-thermal condition on the cylinder surface, $E_{\max }$ and $E_{\text {mean }}$, as below:

$$
\begin{aligned}
E_{\max } & =\max \left\{\left|T_{\mathrm{s}}-T\left(\boldsymbol{X}_{k}\right)\right| ; k=1, \cdots, N\right\} \times 100 \quad[\%] \\
E_{\text {mean }} & =\frac{\sum_{k=1}^{N}\left|T_{\mathrm{s}}-T\left(\boldsymbol{X}_{k}\right)\right|}{N} \times 100 \quad[\%]
\end{aligned}
$$

In addition, we calculate the mean Nusselt number $\overline{N u}$ on the cylinder surface and compare the present result with that by Dennis et al. [30]. The local Nusselt number $N u$ at a point on the cylinder surface with the $\operatorname{argument} \theta$ is defined by

$$
N u(\theta)=\frac{h_{\mathrm{n}}(\theta)}{\lambda_{\mathrm{f}} \Delta T / D_{\mathrm{s}}},
$$

where $h_{\mathrm{n}}(\theta)$ is the heat flux in the normal direction of the cylinder surface at the point and $\Delta T=T_{\mathrm{s}}-T_{\infty}$ is the characteristic temperature difference. The mean Nusselt number $\overline{N u}$ is defined by the mean value of $N u$ on the surface as follows:

$$
\overline{N u}=\frac{1}{\pi D_{\mathrm{s}}} \int_{0}^{2 \pi} N u(\theta) \frac{D_{\mathrm{s}}}{2} d \theta=\frac{1}{\pi D_{\mathrm{s}}} \frac{1}{\lambda_{\mathrm{f}} \Delta T / D_{\mathrm{s}}} \int_{0}^{2 \pi} h_{\mathrm{n}}(\theta) \frac{D_{\mathrm{s}}}{2} d \theta .
$$


The integral in the right-hand side of the above equation should be equal to the rate of total heat transferred from the body to the surrounding fluid given by Eq. (10). Therefore, the mean Nusselt number $\overline{N u}$ can be calculated as follows:

$$
\overline{N u}=\frac{Q}{\pi \lambda_{\mathrm{f}} \Delta T} .
$$

It should be noted that $Q_{\text {in }}=0$ since we consider the steady state, and consequently $Q$ can be calculated by the summation of the heat source/sink term over all lattice points. Table 1 shows $E_{\text {mean }}, E_{\max }$, and $\overline{N u}$ for different numbers of iterations $(m=0,1, \ldots, 5)$ with the result given by Dennis et al. [30]. We can see that the error from the Dirichlet condition is sufficiently small in the case of $m=5$; the maximum error $E_{\max }$ is less than $0.05 \%$ and the mean error $E_{\text {mean }}$ is less than $0.02 \%$. In addition, it can be seen that $\overline{N u}$ is compatible with the result given by Dennis et al. [30].

Table 1: Comparison of $E_{\text {mean }}, E_{\max }$, and $\overline{N u}$ for different numbers of iterations $(m=$ $0,1, \cdots, 5)$. The relative error of $\overline{N u}$ from the result given by Dennis et al. [30] is also shown.

\begin{tabular}{llll}
\hline$m$ & $E_{\text {mean }}[\%]$ & $E_{\max }[\%]$ & $\overline{N u}$ \\
\hline 0 & 0.430 & 0.730 & $2.529(1.10 \%)$ \\
1 & 0.165 & 0.288 & $2.535(0.86 \%)$ \\
2 & 0.0831 & 0.152 & $2.537(0.78 \%)$ \\
3 & 0.0461 & 0.0922 & $2.538(0.74 \%)$ \\
4 & 0.0269 & 0.0625 & $2.538(0.74 \%)$ \\
5 & 0.0161 & 0.0458 & $2.539(0.70 \%)$ \\
& & & \\
\multicolumn{2}{l}{ Dennis et al. [30] } & & 2.557 \\
\hline
\end{tabular}

Next, we compare the local Nusselt number on the cylinder surface with other numerical results $[9,30-32]$. In this simulation, we set $D_{\mathrm{s}}=96 \Delta x$ and $N=332$, which are the same as those in Ref. [9]. In addition, we set $U_{\infty}=0.03986, \tau_{f}=1.074$, and $\tau_{g}=1.286$. Since the local Nusselt number on the cylinder surface cannot be calculated by using the heat source/sink terms 


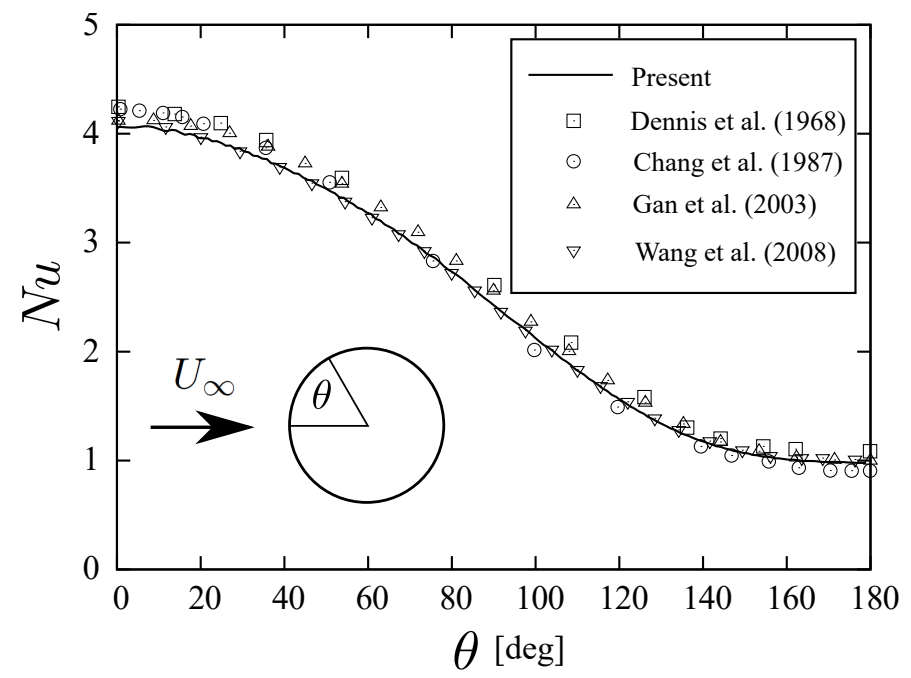

Figure 3: Comparison of the local Nusselt number on the cylinder surface with other numerical results $[9,30-32]$ for the thermal flow around an iso-thermal circular cylinder.

applied near the boundary unlike the mean Nusselt number, we calculate it in a finite difference form near the cylinder surface. It should be noted that the IBM has a problem that the boundary has the effective thickness [34-38], i.e., the diameter of the circular cylinder should be slightly larger than the input diameter $D_{\mathrm{s}}$ effectively. Therefore, we define a virtual cylinder surface which is concentric with and slightly larger than the original circular cylinder, and we regard the virtual cylinder as an effective cylinder. We set the effective thickness of the boundary to $1.25 \Delta x$ [38], i.e., the diameter of the effective cylinder to $D_{\mathrm{s}}+2.5 \Delta x$. We calculate the local heat flux on the effective cylinder surface by the first-order one-sided finite difference approximation of the temperature. Fig. 3 shows the local Nusselt numbers obtained by the present method and other numerical methods $[9,30-32]$. We can see that the present result has good agreement with the other results.

\subsubsection{Taylor-Couette flow with heat transfer for the Dirichlet condition}

We consider a thermal flow between two concentric circular cylinders. Let the axial direction be the $z$-axis and a plane normal to the $z$-axis be the $x$-y 

transfer induced by the difference of the temperatures.

The accuracy of the IB-LBM for the fluid motion (i.e., the velocity, the pressure, and the torque acting on the inner cylinder) was investigated in the previous work [37]. In the present study, therefore, we focus on the accuracy for the heat transfer. The steady solution of the temperature for this problem is given by

$$
T(r)=T_{2}-\left(T_{2}-T_{1}\right) \frac{\ln \left(r / R_{2}\right)}{\ln \left(R_{1} / R_{2}\right)},
$$

where $r$ is the distance from the center of the cylinder. The rate of total heat transferred from the inner cylinder to the surrounding fluid is given by

$$
Q=\frac{2 \pi \lambda_{\mathrm{f}}\left(T_{2}-T_{1}\right)}{\ln \left(R_{1} / R_{2}\right)} .
$$

We take a computational domain of size $[-H, H] \times[-H, H]$ where $H=$ $R_{2}+2 \Delta x$. All sides of the computational domain are periodic. The Reynolds number defined by $R e=U_{1}\left(2 R_{1}\right) / \nu$ is fixed to 10 , and the Prandtl number defined by $\nu / \alpha$ is fixed to 0.73 . The relaxation times are set to $\tau_{f}=0.7880$ and $375 \tau_{g}=0.8945$ in this simulation. The number of Lagrangian boundary points $N$ is set to $4\left(2 R_{1}+1\right)$ for the inner cylinder and $4\left(2 R_{2}+1\right)$ for the outer cylinder.

We calculate errors in the temperature and the rate of total heat from the above analytical solutions. The error norms are defined as follows. The maximum and mean errors in the temperature $T$ are given by

$$
\begin{aligned}
E_{\max }(T) & =\max \left\{\left|T_{\text {calc }}-T_{\text {ex }}\right| ; R_{1}^{2} \leq x^{2}+y^{2} \leq R_{2}^{2}\right\} \\
E_{\text {mean }}(T) & =\frac{1}{M_{\text {in }}} \sum_{R_{1}^{2} \leq x^{2}+y^{2} \leq R_{2}^{2}}\left|T_{\text {calc }}-T_{\text {ex }}\right|
\end{aligned}
$$

where $T_{\text {calc }}$ is a calculated value of $T, T_{\text {ex }}$ is the analytical value of $T, \sum_{R_{1}^{2} \leq x^{2}+y^{2} \leq R_{2}^{2}}$ means the summation over lattice points in the range of $R_{1}^{2} \leq x^{2}+y^{2} \leq R_{2}^{2}$, 

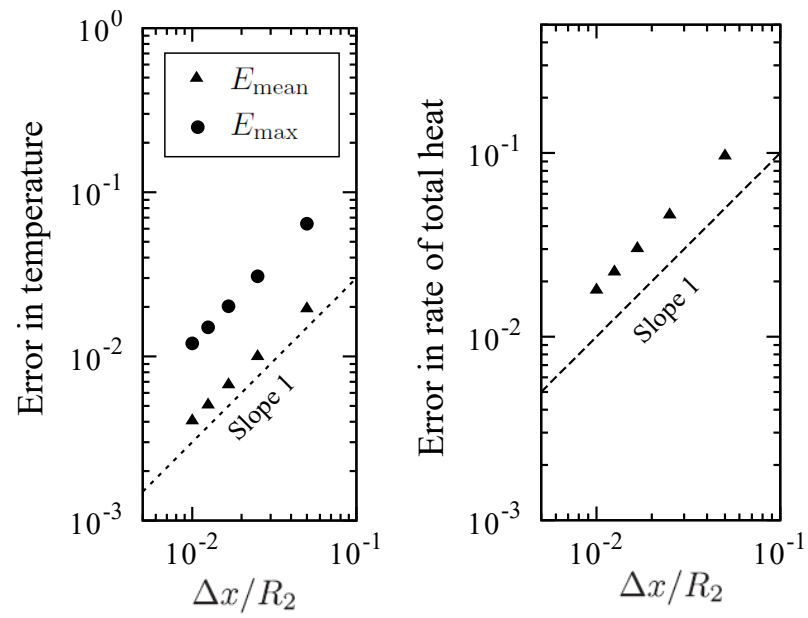

Figure 4: Errors in the temperature and the rate of total heat transferred from the inner cylinder to the surrounding fluid in the simulation of a Taylor-Couette flow with heat transfer for the Dirichlet condition.

and $M_{\mathrm{in}}$ is the number of lattice points in this range. The error in the rate of total heat $Q$ is given by

$$
E(Q)=\left|\frac{Q_{\text {calc }}-Q_{\mathrm{ex}}}{Q_{\mathrm{ex}}}\right|,
$$

where $Q_{\text {calc }}$ is a calculated value of $Q$, and $Q_{\text {ex }}$ is the analytical value of $Q$. The steady state is determined when six significant digits for $E_{\max }(T), E_{\text {mean }}(T)$, and $E(Q)$ converge to constant values. Fig. 4 shows the decay of the errors against the lattice spacing $\Delta x$. We can see from this figure that the present results have first-order accuracy in both the temperature and the rate of total heat. This means that the order of accuracy of the LBM, which is formally second-order accurate, decreases by using the IBM. This is because the discontinuity of the temperature gradient makes a decrease in the order of accuracy of the temperature field in the same way as the fact that the discontinuity of the velocity gradient makes a decrease in the order of accuracy of the flow field $[37,39,40]$. It should be noted that the order of the accuracy is comparable with other thermal IB-LBMs (e.g., [17]). 


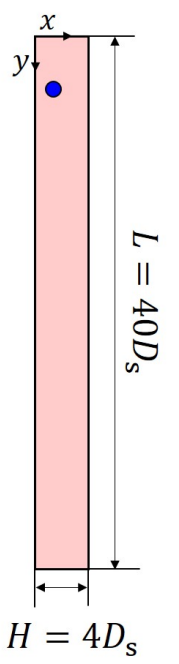

(a)

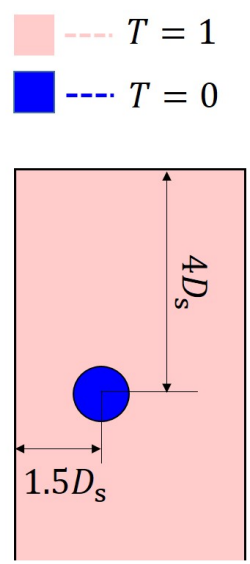

(b)

Figure 5: Computational domain for the sedimentation of a cold circular cylinder in a long channel: (a) the whole domain; (b) the initial position of the circular cylinder.

\subsubsection{Sedimentation of a cold circular cylinder in a long channel}

We consider the sedimentation of a cold circular cylinder at a constant temperature in a long channel. This problem has been numerically investigated and regarded as a good benchmark problem by many researchers $[8,13,15,17$, $32,41-43]$. In this study, we compare the present results with recent results calculated by Eshghinejadfard and Thévenin [15].

The computational domain and the coordinate system are shown in Fig. 5. The diameter of the circular cylinder is $D_{\mathrm{s}}$, and the size of the domain is $H \times L=$ $4 D_{\mathrm{s}} \times 40 D_{\mathrm{s}}$. The fluid is initially at rest and at a temperature $T_{\mathrm{f}}=1$. The circular cylinder is at a constant temperature $T_{\mathrm{s}}=0$ and the initial position of its center is $\left(X_{\mathrm{c}}, Y_{\mathrm{c}}\right)=\left(1.5 D_{\mathrm{s}}, 4 D_{\mathrm{s}}\right)$, i.e., deviated by $0.5 D_{\mathrm{s}}$ from the center of the channel (Fig. 5b). The circular cylinder falls under gravity with the gravitational acceleration $\alpha_{\mathrm{g}}$. Letting the density of the circular cylinder be $\rho_{\mathrm{s}}$, the mass and the inertia moment of the circular cylinder are $M=\rho_{\mathrm{s}}\left(\pi D_{\mathrm{s}}^{2} / 4\right)$ and $I_{\mathrm{B}}=M D_{\mathrm{s}}^{2} / 8$, respectively. The net gravitational force is $F_{\mathrm{g}}=\left(1-\rho_{\mathrm{f}} / \rho_{\mathrm{s}}\right) M \alpha_{\mathrm{g}}$, and the buoyancy force $F_{\mathrm{b}}$ by thermal expansion of the fluid is given by the 
Boussinesq approximation, i.e.,

$$
F_{\mathrm{b}}(\boldsymbol{x}, t)=\rho_{\mathrm{f}} \beta \alpha_{\mathrm{g}}\left(T(\boldsymbol{x}, t)-T_{\mathrm{f}}\right)
$$

395

can give an accurate result for moving-boundary flows where the fluid motion, the body motion, and the heat transfer interact with each other. 


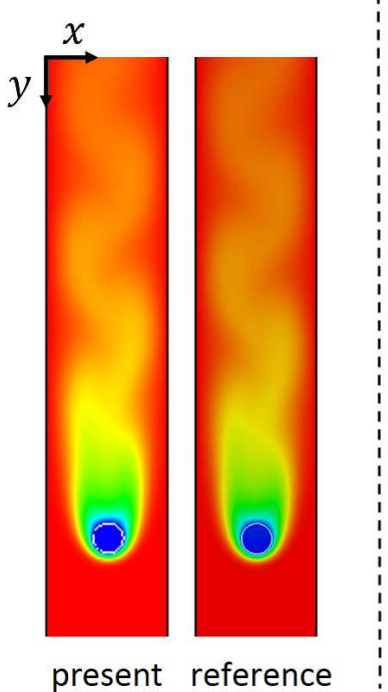

(a)

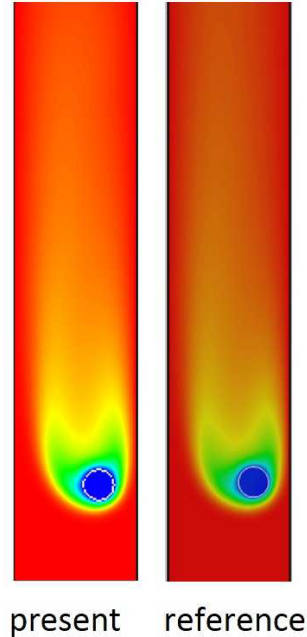

(b)

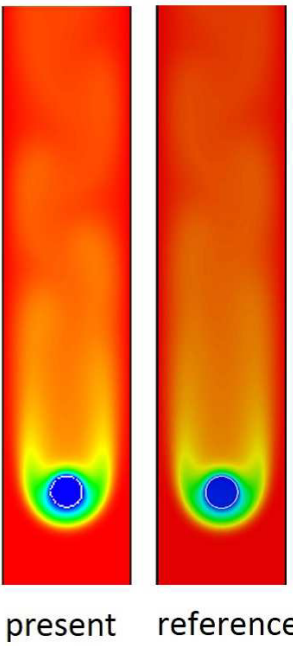

(c)

Temperature

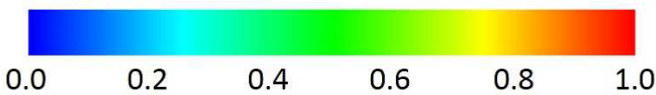

Figure 6: Snapshots of the temperature fields around the circular cylinder for (a) $G r=564$,(b) 2000, and (c) 4500. The results by Eshghinejadfard and Thévenin [15] are also shown as a reference.

\subsection{For the Neumann condition}

\subsubsection{Thermal flow around an iso-heat-flux circular cylinder}

We consider a thermal flow around an iso-heat-flux circular cylinder. The diameter of the circular cylinder is $D_{\mathrm{s}}$. The computational domain is $\left[-14 D_{\mathrm{s}}, 20 D_{\mathrm{s}}\right] \times$ $\left[-15 D_{\mathrm{s}}, 15 D_{\mathrm{s}}\right]$. The center of the circular cylinder is located at $(x, y)=(0,0)$. In the inlet $\left(x=-14 D_{\mathrm{s}}\right)$, a uniform iso-thermal flow in the $x$-direction with a speed $U_{\infty}$ and at a temperature $T_{\infty}=0$ is imposed. The other boundary conditions are the same as those in Section 4.1.1. The circular cylinder is stationary and iso-heat-flux with a constant heat flux $H^{\mathrm{d}}$, whose nondimensional value $H^{\mathrm{d} *}=H^{\mathrm{d}} /\left(\lambda_{\mathrm{f}} \Delta T / D_{\mathrm{s}}\right)$ is fixed to 1 . The governing parameters of this system are the Reynolds number defined by $R e=U_{\infty} D_{\mathrm{s}} / \nu$ and the Prandtl number 


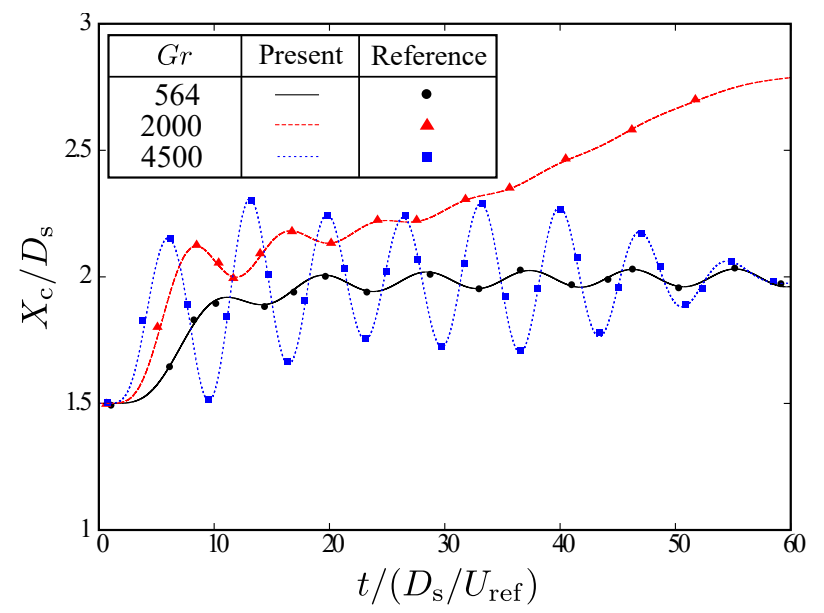

Figure 7: Trajectories of the center of the circular cylinder for $G r=564,2000$, and 4500 . The results by Eshghinejadfard and Thévenin [15] are also shown as a reference.

defined by $\operatorname{Pr}=\nu / \alpha$. We set $R e=10,20$, and 40 and $\operatorname{Pr}=0.7$ in order to compare the present results with other numerical results $[10,18,44]$. We consider the steady state of this system. The steady state is determined when four significant digits for the mean Nusselt number $\overline{\mathrm{Nu}}$ (shown below) converge to constant values. In the present simulation, we set $D_{\mathrm{s}}=40 \Delta x, N=164$, $U_{\infty}=0.04$, and $\Delta T=1$.

Fig. 8 shows isotherms around the circular cylinder in the range of $\left[-D_{\mathrm{s}}, 4.015 D_{\mathrm{s}}\right] \times$ $\left[-1.65 D_{\mathrm{s}}, 1.65 D_{\mathrm{s}}\right]$. In this figure, the results by Wang et al. [18] (Fig. 9 in this reference) are also shown for comparison. From this figure, we can see that the present results reasonably agree with the results by Wang et al. [18].

Next, we calculate the local Nusselt number on the cylinder surface defined by

$$
N u(\theta)=\frac{H^{\mathrm{d}}}{\lambda_{\mathrm{f}} T(\theta) / D_{\mathrm{s}}},
$$

where $T(\theta)$ is the temperature on the cylinder surface at the argument $\theta$, and it is calculated by interpolating the temperature from the neighboring lattice points as shown in Eq. (37). Fig. 9 shows the local Nusselt numbers on the cylinder surface obtained by the present method and other numerical methods $[18,44]$. 
We can see from this figure that the present results have good agreement with other results for all the cases of $R e=10,20$, and 40 . Table 2 shows the mean Nusselt number $\overline{N u}$ obtained by integrating the local Nusselt number. From this table, it can be seen that even for the mean Nusselt number the present results have good agreement with other results [10, 18, 44].

\subsubsection{Taylor-Couette flow with heat transfer for the Neumann condition}

We consider the Taylor-Couette flow with heat transfer for the Neumann condition. The computational condition is almost the same as that in Sec-

(a)
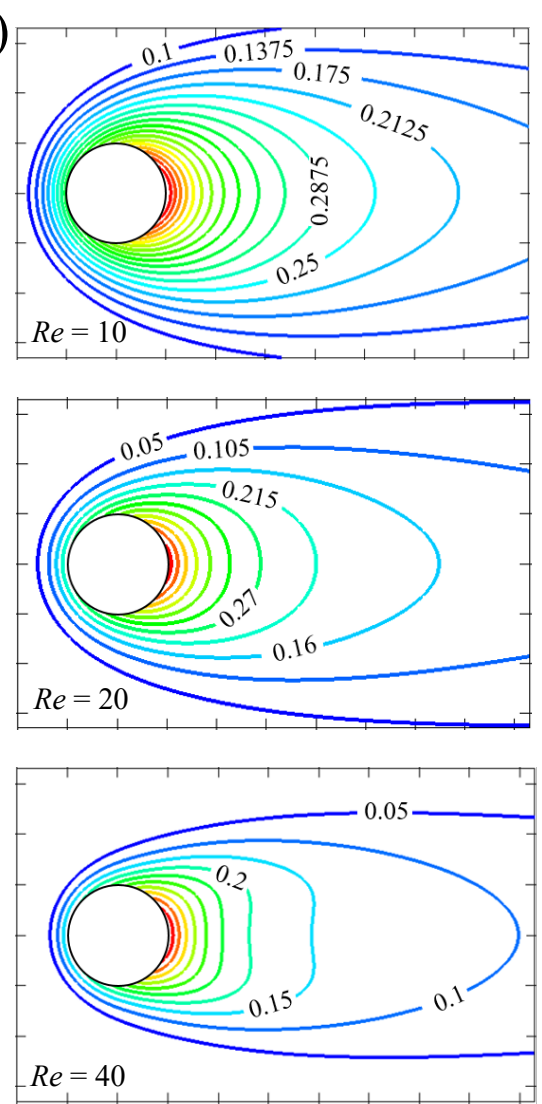

(b)
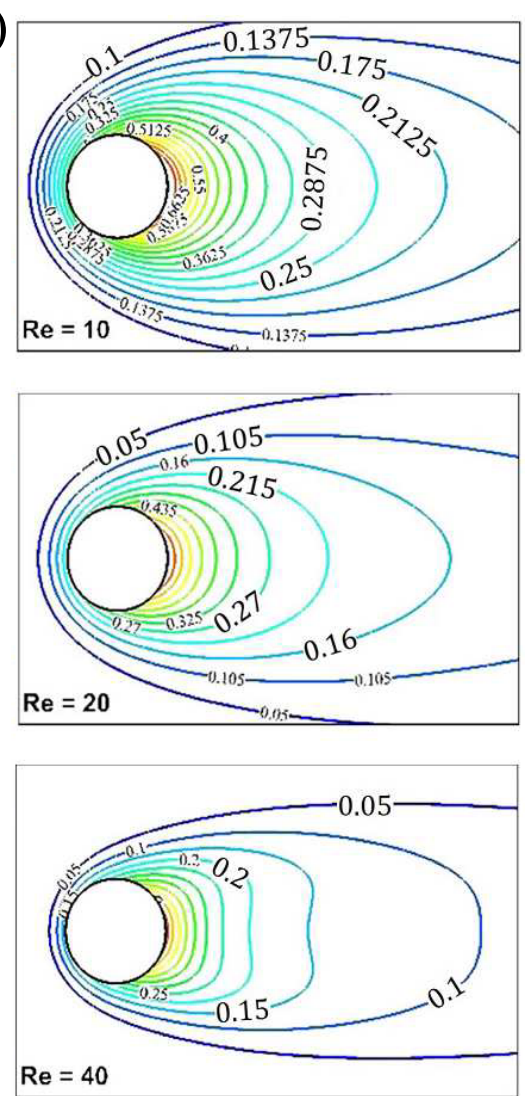

Figure 8: Comparison of isotherms around the circular cylinder for the thermal flow around an iso-heat-flux circular cylinder: (a) the present results; (b) numerical results by Wang et al. [18]. 


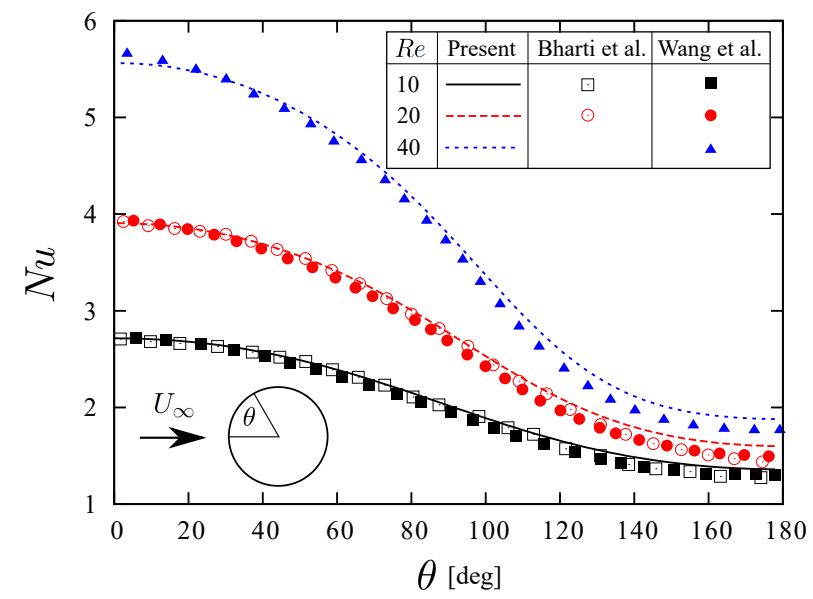

Figure 9: Comparison of the local Nusselt number on the cylinder surface with other numerical results by Bharti et al. [44] and by Wang et al. [18] for the thermal flow around an iso-heat-flux circular cylinder.

Table 2: Comparison of the mean Nusselt number $\overline{N u}$ with other numerical results $[10,18,44]$.

\begin{tabular}{ccccc}
\hline \multirow{2}{*}{$R e$} & $\overline{N u}$ & & & \\
\cline { 2 - 5 } & Present & Bharti et al. [44] & Ren et al. [10] & Wang et al. [18] \\
\hline 10 & 2.028 & 2.040 & 2.016 & 2.01 \\
20 & 2.753 & 2.779 & 2.741 & 2.69 \\
40 & 3.719 & 3.775 & 3.741 & 3.68 \\
\hline
\end{tabular}

tion 4.1.2 except that the inner cylinder is heated with a constant heat flux $H^{\mathrm{d}}$. In this calculation, supposing that the nondimensional heat flux $H^{\mathrm{d} *}=$ $H^{\mathrm{d}} /\left(\lambda_{\mathrm{f}} \Delta T / R_{2}\right)$ is fixed to 1 (where $\Delta T=1$ in this simulation), we consider a heat transfer induced by the heat flux. The other conditions are the same as those in Section 4.1.2.

For the same reason explained in Section 4.1.2, we focus on the accuracy for the heat transfer. The steady solution of the temperature for this problem is given by

$$
T(r)=\frac{H^{\mathrm{d}}}{\lambda_{\mathrm{f}}} R_{1} \ln \left(\frac{R_{2}}{r}\right)
$$


where $r$ is the distance from the center of the cylinder. The rate of total heat transferred from the inner cylinder to the surrounding fluid is given by

$$
Q=2 \pi R_{1} H^{\mathrm{d}}
$$

We calculate errors in the temperature and the rate of total heat from the above analytical solutions. The maximum and mean errors in the temperature $T$ are given by

$$
\begin{aligned}
E_{\text {max }}(T) & =\frac{\max \left\{\left|T_{\text {calc }}-T_{\text {ex }}\right| ; R_{1}^{2} \leq x^{2}+y^{2} \leq R_{2}^{2}\right\}}{H^{\mathrm{d}} R_{2} / \lambda_{\mathrm{f}}}, \\
E_{\text {mean }}(T) & =\frac{1}{M_{\text {in }}} \frac{\sum_{R_{1}^{2} \leq x^{2}+y^{2} \leq R_{2}^{2}}\left|T_{\text {calc }}-T_{\text {ex }}\right|}{H^{\mathrm{d}} R_{2} / \lambda_{\mathrm{f}}},
\end{aligned}
$$

where $T_{\text {calc }}$ is a calculated value of $T$, and $T_{\text {ex }}$ is the analytical value of $T$. The error norm for $Q$ is the same as that used in Section 4.1.2. The steady state is determined when six significant digits for $E_{\max }(T), E_{\text {mean }}(T)$, and $E(Q)$ converge to constant values. Fig. 10 shows the decay of the errors for the lattice spacing $\Delta x$. We can see from this figure that the present results have first-order accuracy in both the temperature and the rate of total heat. The decrease in the order of accuracy compared with that of the LBM can be explained in the same way as the case of the Dirichlet condition (see Section 4.1.2).

\subsubsection{Natural convection in an annulus}

We consider a natural convection in an annuls, which has been studied in many researches $[10,17,18,45]$. Let the axial direction be the $z$-axis and a plane normal to the $z$-axis be the $x$-y plane. The radius of the inner cylinder is $R_{1}$ and that of the outer cylinder is $R_{2}=2 R_{1}$. The inner and outer cylinders are stationary. The inner cylinder is heated with a constant heat flux $H^{\mathrm{d}}$,

475 and the outer cylinder is iso-thermal at a constant temperature $T_{\mathrm{f}}=0$. In this calculation, supposing that the nondimensional heat flux $H^{\mathrm{d} *}=H^{\mathrm{d}} /\left(\lambda_{\mathrm{f}} \Delta T / R_{2}\right)$ is fixed to 1 (where $\Delta T=1$ in this simulation), we consider a flow with heat transfer induced by the buoyancy force $F_{\mathrm{b}}$ by thermal expansion of the fluid given by Eq. (58). 

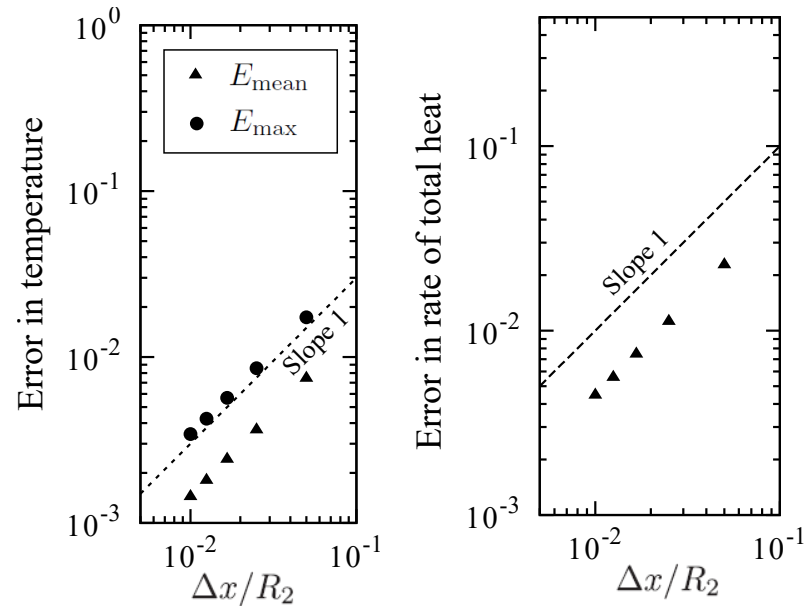

Figure 10: Errors in the temperature and the rate of total heat transferred from the inner cylinder to the surrounding fluid in the simulation of a Taylor-Couette flow with heat transfer for the Neumann condition.

We take a computational domain of size $[-H, H] \times[-H, H]$ where $H=R_{2}+$ $2 \Delta x$. The buoyancy force $F_{\mathrm{b}}$ is applied only in the area between the inner and outer cylinders in the $y$-direction. In all sides of the computational domain, the no-slip condition and the iso-thermal condition at a constant temperature $T_{\mathrm{f}}=0$ are imposed by the bounce-back scheme [33] and the iso-thermal condition [21]. The Rayleigh number defined by $R a=\beta \alpha_{\mathrm{g}} L^{4} H^{\mathrm{d}} /\left(\lambda_{\mathrm{f}} \alpha \nu\right)$ (where $L=R_{2}-R_{1}$ ) is fixed to 5700, and the Prandtl number defined by $\nu / \alpha$ is fixed to 0.7 . The relaxation times are set to $\tau_{f}=0.7880$ and $\tau_{g}=0.8945$ in this simulation. The number of Lagrangian boundary points $N$ is set to $4\left(2 R_{1}+1\right)$ for the inner cylinder and $4\left(2 R_{2}+1\right)$ for the outer cylinder. We define the convergence criterion for this problem by using the maximum differences of the flow velocity and the temperature as used in Ref. [46], i.e.,

$$
\begin{aligned}
& \max \left\{\left|\sqrt{\left(u^{n+1}\right)^{2}+\left(v^{n+1}\right)^{2}}-\sqrt{\left(u^{n}\right)^{2}+\left(v^{n}\right)^{2}}\right| ; R_{1}^{2} \leq x^{2}+y^{2} \leq R_{2}^{2}\right\} \leq 10^{-8} \\
& \max \left\{\left|T^{n+1}-T^{n}\right| ; R_{1}^{2} \leq x^{2}+y^{2} \leq R_{2}^{2}\right\} \leq 10^{-8}
\end{aligned}
$$


Fig. 11(a) shows the temperature on the cylinder surface in the steady state obtained by the present method and other numerical methods $[10,18,45]$. In the present result, we set $R_{1}=50 \Delta x$. We can see from this figure that the present result reasonably agrees with the other numerical results.

In addition, we calculate the temperature field for six different grid sizes of $R_{1}=10 \Delta x, 12.5 \Delta x, 20 \Delta x, 25 \Delta x, 50 \Delta x$, and $100 \Delta x$, and we examine the order of accuracy when the result of the finest grid is taken as the reference solution. Note that the analytical solution of this problem is unknown. Fig. 11(b) shows the $L_{2}$ norm of the temperature and the order of accuracy, where the $L_{2}$ norm is defined by

$$
L_{2}(T)=\sqrt{\frac{\sum_{R_{1}^{2} \leq x^{2}+y^{2} \leq R_{2}^{2}}\left|T_{\text {calc }}-T_{\text {ref }}\right|^{2}}{\sum_{R_{1}^{2} \leq x^{2}+y^{2} \leq R_{2}^{2}}\left|T_{\text {ref }}\right|^{2}}},
$$

485 accuracy in the iso-heat-flux condition for the present method is inferior to that for their method. However, it should be noted that the present method can 

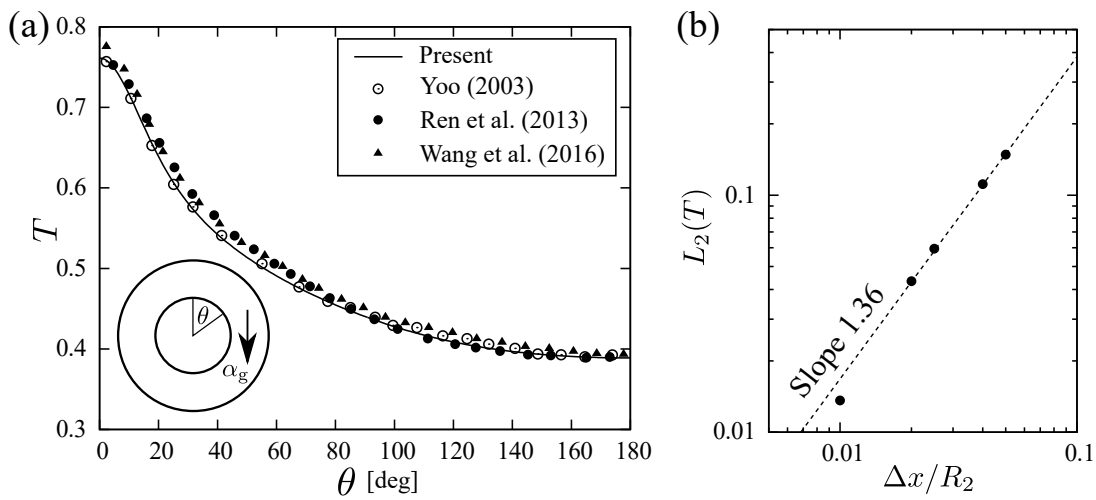

Figure 11: (a) Comparison of temperature profile on the inner cylinder surface for natural convection in an annulus at $R a=5700$, and (b) the $L_{2}$ norm of the temperature and the order of accuracy when the result of the finest grid is taken as the reference solution.

calculate the temperature field accurately as shown in the previous sections, although the temperature gradient around the boundary is not accurate. In addition, the present method is easily applied to moving-boundary flows without increasing the computational cost, while the method of Wang et al. [18] has not been applied to moving-boundary flows and its computational cost might increase in moving-boundary flows due to calculations of the geometry-related matrices and their inversions in every time step. Therefore, we believe that the present method is advantageous for moving-boundary flows in terms of the computational efficiency.

\subsubsection{Heat convection with flow over an oscillating circular cylinder with a} constant heat flux

We consider a heat convection with flow over an oscillating circular cylinder with a constant heat flux. The diameter of the circular cylinder is $D_{\mathrm{s}}$. The computational domain is $\left[-6.4 D_{\mathrm{s}}, 19.2 D_{\mathrm{s}}\right] \times\left[-6.4 D_{\mathrm{s}}, 6.4 D_{\mathrm{s}}\right]$. The center $\boldsymbol{X}_{\mathrm{c}}=$ $\left(x_{\mathrm{c}}, y_{\mathrm{c}}\right)$ of the circular cylinder oscillates in the $y$-direction as follows:

$$
\begin{aligned}
& x_{\mathrm{c}}(t)=0, \\
& y_{\mathrm{c}}(t)=A \sin (2 \pi f t),
\end{aligned}
$$




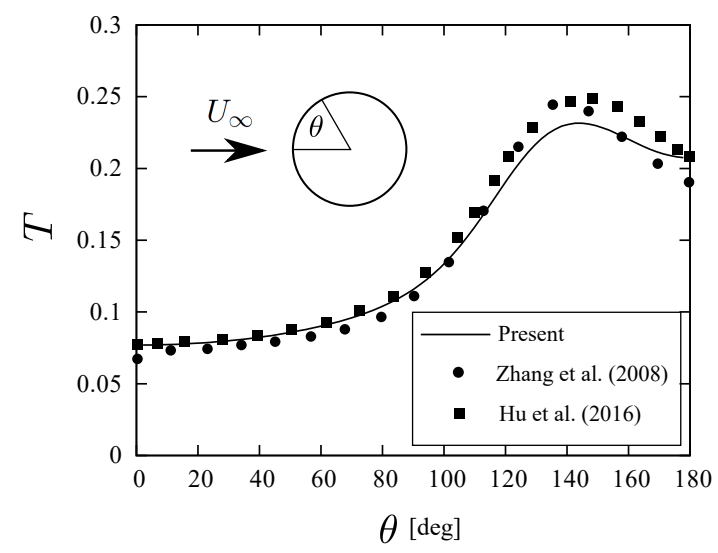

Figure 12: Comparison of the time-averaged temperature on the cylinder surface with other numerical results by Zhang et al. [7] and by $\mathrm{Hu}$ et al. [17] for the flow over an oscillating circular cylinder with a constant heat flux.

where $A$ is the amplitude of the position and $f$ is the frequency. In the inlet ( $x=$ $\left.-6.4 D_{\mathrm{s}}\right)$, a uniform iso-thermal flow in the $x$-direction with a speed $U_{\infty}$ and at a temperature $T_{\infty}=0$ is imposed. The other boundary conditions are the same as those in Section 4.1.1. The circular cylinder is iso-heat-flux with a constant heat flux $H^{\mathrm{d}}$, whose nondimensional value $H^{\mathrm{d} *}=H^{\mathrm{d}} /\left(\lambda_{\mathrm{f}} \Delta T / D_{\mathrm{s}}\right)$ is fixed to 1 . $R e=U_{\infty} D_{\mathrm{s}} / \nu$, the Prandtl number defined by $\operatorname{Pr}=\nu / \alpha$, the Strouhal number defined by $S t=f D_{\mathrm{s}} / U_{\infty}$, and the Keulegan-Carpenter number defined by $K C=2 \pi A / D_{\mathrm{s}}$. We set $R e=200, P r=0.7, S t=0.2$, and $K C=0.3 \pi$ in order to compare the present results with other numerical results $[7,17]$. In the present simulation, we set $D_{\mathrm{s}}=40 \Delta x, N=164, U_{\infty}=0.04$, and $\Delta T=1$.

Fig. 12 shows the time-averaged temperature on the cylinder surface obtained by the present method and other numerical methods $[7,17]$. We can see from this figure that the present result reasonably agrees with other numerical results. This means that the present thermal IB-LBM can give a reasonable result for moving-boundary flows with heat transfer for the iso-heat-flux condition. 
4.3. Internal heat effect in the simulation of moving-boundary flows with heat transfer by the thermal IBM

In this section, we investigate the internal heat effect $Q_{\text {in }}$ given by Eq. (12)

535

fluid. Since the internal mass effect $\boldsymbol{F}_{\text {in }}$ and $\boldsymbol{T}_{\text {in }}$ for the force and torque depend on the Reynolds number as investigated by Suzuki and Inamuro [19], it can be expected that the internal heat effect $Q_{\text {in }}$ for the rate of total heat depends on the Reynolds number and the Péclet number. Therefore, we investigate simulations of moving-boundary flows with heat transfer by the present thermal IB-LBM. Unfortunately, however, we could not find appropriate reference data about the rate of total heat in moving-boundary flows with heat transfer. Hence, we construct a new benchmark problem for investigating the internal heat effect $Q_{\text {in }}$ for the rate of total heat.

\subsubsection{Problem and computational condition}

We consider a heated circular cylinder which oscillates translationally in a closed small box at a low temperature. The diameter of the circular cylinder is $D_{\mathrm{s}}$. The computational domain is $\left[-3 D_{\mathrm{s}}, 3 D_{\mathrm{s}}\right] \times\left[-2 D_{\mathrm{s}}, 2 D_{\mathrm{s}}\right]$. The cylinder placed at the center of the domain suddenly starts to oscillate in the $x$-direction

Table 3: The parameters used in simulations of Section 4.3.

\begin{tabular}{rrrrrr}
\hline$R e$ & $P e$ & $K C$ & $U_{\max }$ & $\tau_{f}$ & $\tau_{g}$ \\
\hline 100 & 100 & 5 & 0.03 & 0.545 & 0.545 \\
10 & 100 & 5 & 0.03 & 0.950 & 0.545 \\
1 & 100 & 5 & 0.03 & 5.00 & 0.545 \\
100 & 10 & 5 & 0.03 & 0.545 & 0.950 \\
100 & 1 & 5 & 0.03 & 0.545 & 5.00 \\
\hline
\end{tabular}


with the following speed:

$$
\begin{aligned}
& U_{\mathrm{c}}(t)=U_{\max } \cos (2 \pi f t), \\
& V_{\mathrm{c}}(t)=0,
\end{aligned}
$$

where $U_{\mathrm{c}}$ and $V_{\mathrm{c}}$ are the respective velocity components in the $x$ - and $y$-directions of the cylinder, $U_{\max }$ is the amplitude of the speed, and $f$ is the frequency. All the walls of the box are stationary and iso-thermal at a constant temperature $T_{\mathrm{w}}=0$, and the boundary conditions are implemented by the bounce-back scheme [33] and the iso-thermal condition [21]. The fluid is initially at rest and at a temperature $T_{\mathrm{w}}=0$. In this study, we consider two cases where the circular cylinder is iso-thermal at a constant temperature $T_{\mathrm{s}}=1$ and where it is iso-heat-flux with a constant heat flux $H^{\mathrm{d}}$ (whose nondimensional value $H^{\mathrm{d} *}=H^{\mathrm{d}} /\left(\lambda_{\mathrm{f}} \Delta T / D_{\mathrm{s}}\right)$ is fixed to 1$)$. The governing parameters of this system are the Reynolds number defined by $R e=U_{\max } D_{\mathrm{s}} / \nu$, the Péclet number defined by $P e=U_{\max } D_{\mathrm{s}} / \alpha$, and the Keulegan-Carpenter number defined by $K C=2 \pi A / D_{\mathrm{s}}$ (where $A$ is the amplitude of the position of the cylinder). It should be noted that this problem is an original problem of this study, and no published experimental and numerical data are available. This problem is constructed for effective investigation into the internal heat effect $Q_{\text {in }}$ for various $R e$ and $P e$. In the present simulation, we set $D_{\mathrm{s}}=50 \Delta x, N=204$, and $\Delta T=1$, and other parameters for various cases are shown in Table 3.

\subsubsection{Calculation of the internal heat effect}

The rate of total heat $Q$ transferred from the circular cylinder to the surrounding fluid is calculated by Eq. (10). The summation of the heat source/sink term $Q_{\text {tot }}$ can be calculated as follows:

$$
Q_{\mathrm{tot}}(t)=\sum_{\boldsymbol{x}} q(\boldsymbol{x}, t)(\Delta x)^{2} .
$$

565 In this study, we consider two approximations for calculating the internal heat effect $Q_{\text {in }}$, i.e., (A) No internal heat effect and (B) Lagrangian points approximation, in a similar way to the work by Suzuki and Inamuro [19]. 


\section{(A) No internal heat effect}

The internal heat effect is ignored, that is,

$$
Q_{\text {in }}(t) \approx 0 \text {. }
$$

\section{(B) Lagrangian points approximation}

The most straightforward approximation of Eq. (12) is the finite difference approximation in time for the sum of the specific energy of the internal mass over internal Lagrangian points $\boldsymbol{X}_{\text {in }}(t)$ which move together with the body motion. It should be noted that the temperature $T\left(\boldsymbol{X}_{\mathrm{in}}, t\right)$ on internal Lagrangian points $\boldsymbol{X}_{\text {in }}(t)$ must be interpolated from neighbor lattice points. The interpolation can be implemented by

$$
T\left(\boldsymbol{X}_{\text {in }}, t\right)=\sum_{\boldsymbol{x}} T(\boldsymbol{x}, t) W\left(\boldsymbol{x}-\boldsymbol{X}_{\text {in }}(t)\right)(\Delta x)^{2} .
$$

The total energy $E_{\text {in }}(t)$ of the internal mass at time $t$ is calculated by

$$
E_{\mathrm{in}}(t)=\sum_{\text {all } \boldsymbol{X}_{\mathrm{in}}(t)} \rho_{\mathrm{f}} c_{\mathrm{pf}} T\left(\boldsymbol{X}_{\mathrm{in}}, t\right) \Delta V_{\mathrm{in}},
$$

where $\Delta V_{\text {in }}$ is the volume element of internal Lagrangian points. In the present work, we arrange initial internal Lagrangian points $\boldsymbol{X}_{\text {in }}(0)$ in lattice points with the width of $\Delta x$, and therefore we take $\Delta V_{\text {in }}=(\Delta x)^{2}$ in the same way as the work by Suzuki and Inamuro [19]. The time derivative in $E_{\text {in }}(t)$ is approximated by the change between two successive time $t-\Delta t$ and $t$ as below:

$$
Q_{\mathrm{in}}(t) \approx S h \frac{E_{\mathrm{in}}(t)-E_{\mathrm{in}}(t-\Delta t)}{\Delta t},
$$

where at $t=0$ we assume that $E_{\text {in }}(-\Delta t)=E_{\text {in }}(0)$.

In this study, we investigate the internal heat effect $Q_{\text {in }}$ by comparing the results of (A) and (B) for iso-thermal and iso-heat-flux conditions. 


\subsubsection{Case for iso-thermal condition}

At first, we show the results of the case where the circular cylinder is isovery high frequency in the calculation of $Q_{\text {in }}$ at $P e=100$, the magnitude of 

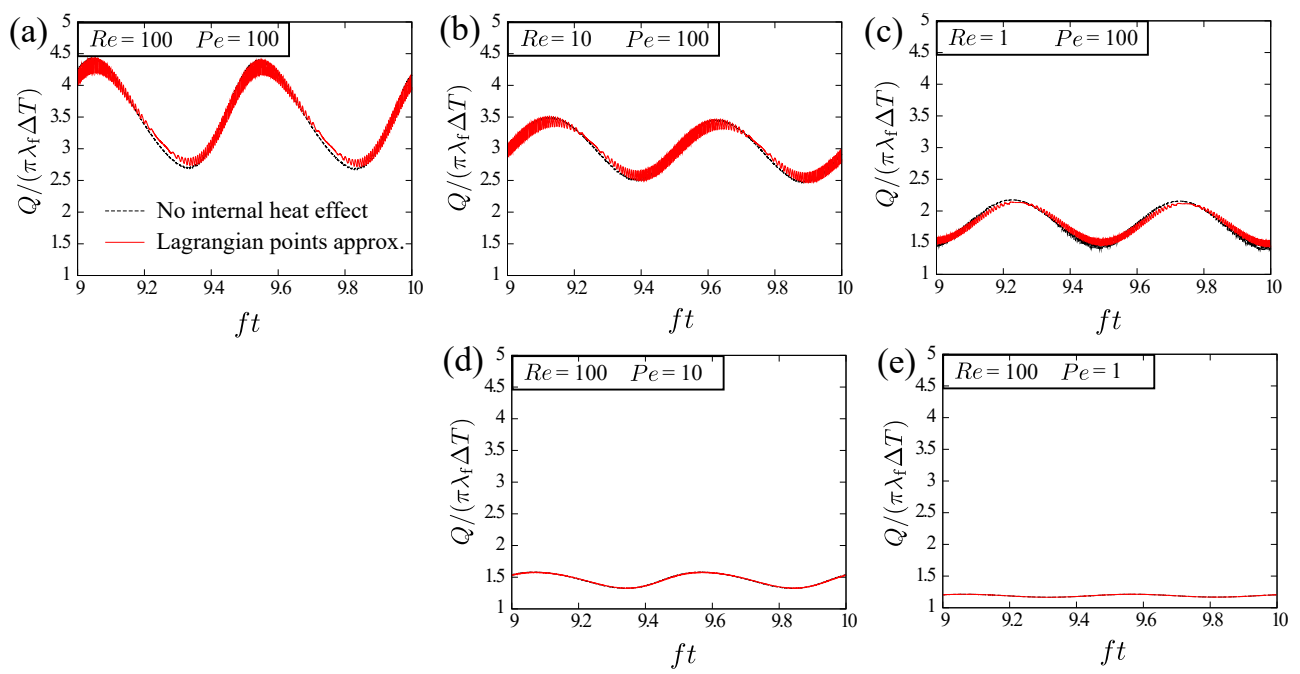

Figure 13: Time variations of the rate of total heat transferred from the circular cylinder to the surrounding fluid for (a) $(R e, P e)=(100,100)$, (b) $(10,100),($ c) $(1,100)$, (d) $(100,10)$, and $(\mathrm{e})(100,1)$ in the case where the circular cylinder is iso-thermal.

the unphysical oscillation decreases as $P e$ decreases, and it becomes negligibly small for $P e \leq 10$. The former conclusion is reasonable as discussed above. Therefore, there is no need to care about the internal heat effect for the isothermal condition with a constant temperature. However, it should be noted that the internal heat effect should be considered for the iso-thermal condition with a space- and/or time-dependent temperature. The latter conclusion shows a serious problem of Lagrangian points approximation at a high Péclet number. The remedy of the problem remains in future work.

\subsubsection{Case for iso-heat-flux condition}

Next, we show the results of the case where the circular cylinder is iso-heatflux with a constant heat flux $H^{\mathrm{d}}$. It should be noted that in this case the analytical solution is available and $Q /\left(\pi \lambda_{\mathrm{f}} \Delta T\right)$ must be equal to 1 .

Fig. 14 shows the time variations of $Q$ for various Reynolds and Péclet numbers in the 10th period of the oscillation $(9 \leq f t \leq 10)$. By comparing Figs. 14(a), (b), and (c), we can find that the results of No internal heat effect 
have a distinct error from the analytical solution $Q /\left(\pi \lambda_{\mathrm{f}} \Delta T\right)=1$ at $P e=100$ independently of $R e$. On the other hand, the deviation between the analytical solution and the results of Lagrangian points approximation is relatively small, although the results of Lagrangian points approximation have unphysical oscillations with very high frequency. These results mean that the magnitude of the internal heat effect is significant at $P e=100$ in the case for the iso-heat-flux condition, and Lagrangian points approximation has a problem in the calculation of $Q_{\text {in }}$, which is the same as in the case for the iso-thermal condition. By comparing Figs. 14(a), (d), and (e), we can see that the error in the results of No internal heat effect from the analytical solution decreases as $P e$ decreases, and it becomes negligibly small for $P e=1$.

In conclusion of the case for the iso-heat-flux condition, the internal heat effect is significant in the range of $10 \leq P e \leq 100$ independently of $R e$, and Lagrangian points approximation gives an unphysical oscillation with very high frequency in the calculation of $Q_{\text {in }}$ at a high Péclet number. The former conclusion shows that the internal heat effect should be considered for $P e \geq 10$ in the case for the iso-heat-flux condition. The latter conclusion is the same as that in the case for the iso-thermal condition.

\section{Application: two-dimensional thermal flow in a heated channel with moving cold particles}

In this section, we apply the present method to an interesting application inspired from ice slurry flow [23]. Ice slurry is a homogenous mixture of small ice particles and carrier liquid, and it can transport cold thermal energy directly because of its fluidity and have a high heat exchange rate because of fine ice particles. However, it is difficult to know the detailed behavior of ice slurry flow in pipes, and therefore it has been seen as a challenge to its successful commercial implementation [47]. In this study, we consider a simplified model of ice slurry flow in a pipe heated by a thermal load, i.e., a two-dimensional thermal flow in a heated channel with moving cold particles. 

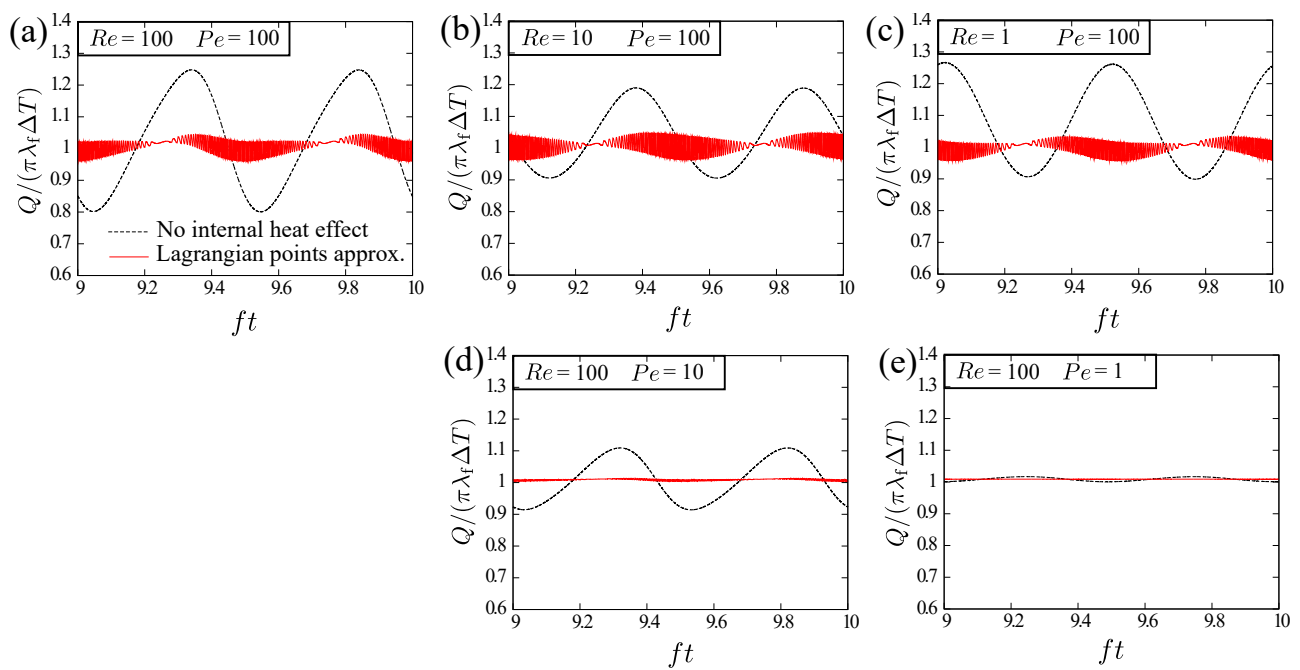

Figure 14: Time variations of the rate of total heat transferred from the circular cylinder to the surrounding fluid for (a) $(R e, P e)=(100,100)$, (b) $(10,100),(c)(1,100),(d)(100,10)$, and $(e)(100,1)$ in the case where the circular cylinder is iso-heat-flux.

\subsection{Modeled system and computational conditions}

We consider a two-dimensional infinitely long channel with width $H=7.5$ $\mathrm{mm}$ as a modeled system of the experiment by Kumano et al. [48]. Ethanol

solution (5 wt.\% concentration) is filled in the channel as the fluid, and the density is $\rho_{\mathrm{f}}=999.78 \mathrm{~kg} / \mathrm{m}^{3}$, the kinematic viscosity is $\nu=2.565 \times 10^{-6}$ $\mathrm{m}^{2} / \mathrm{s}$, and the thermal diffusivity is $\alpha=1.333 \times 10^{-7} \mathrm{~m}^{2} / \mathrm{s}$ (which are for the solidifying temperature $-2^{\circ} \mathrm{C}$ of the ethanol solution [48]). We assume ice particles in the ethanol solution as circular cylinders with density $\rho_{\mathrm{s}}=\rho_{\mathrm{f}}$, i.e., naturally buoyant. A single particle with diameter $D_{\mathrm{s}}$ is contained in every length $L$ of the channel (see Fig. 15). The mass and the inertia moment of the particle are $M=\rho_{\mathrm{s}}\left(\pi D_{\mathrm{s}}^{2} / 4\right)$ and $I_{\mathrm{B}}=M D_{\mathrm{s}}^{2} / 8$, respectively. The channel walls are heated at a constant temperature $T_{\mathrm{w}}=1$, while the particle is at a constant temperature $T_{\mathrm{s}}=0$. We consider a flow induced by a pressure gradient in the $x$-direction, and a heat transfer induced by the temperature difference between the walls and the particles. Melting and solidification of the particle and natural convection are neglected in this study. 


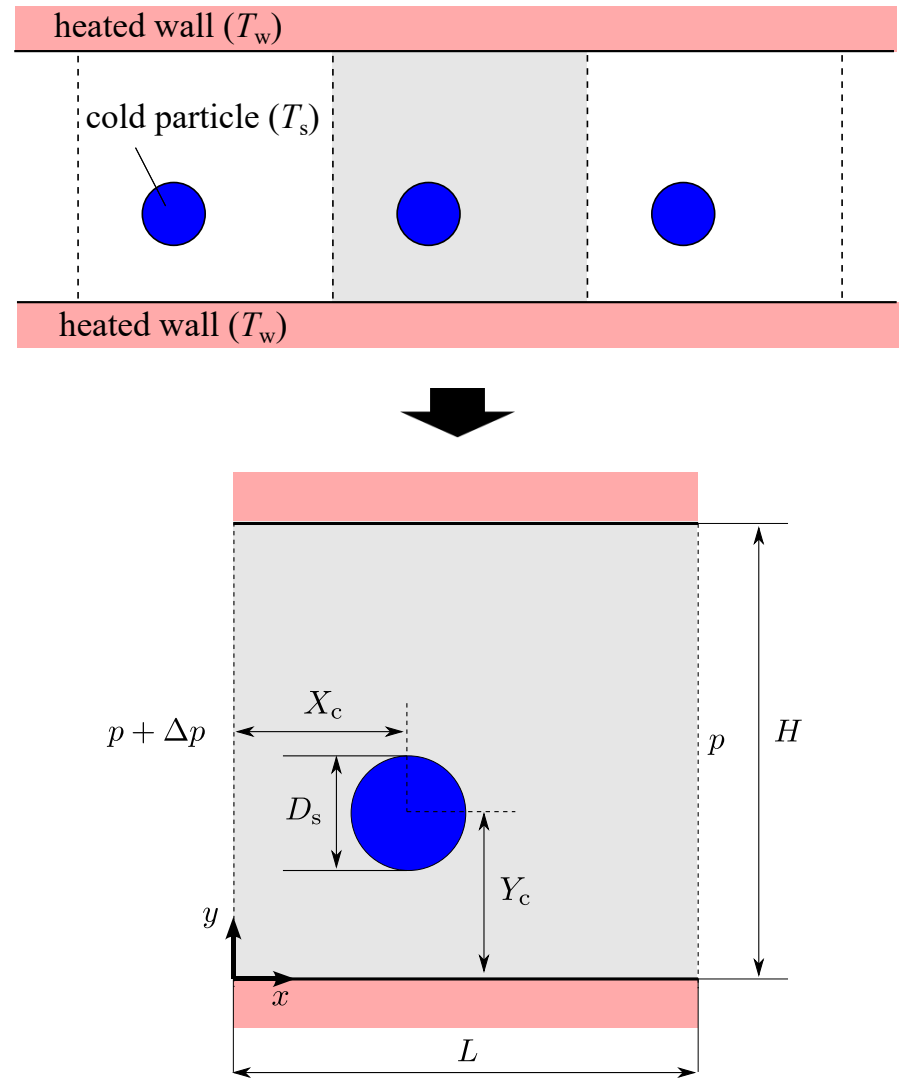

Figure 15: Computational domain for a thermal flow in a heated channel with moving cold particles. The periodic boundary condition with a constant pressure difference $\Delta p$ is used at the inlet and outlet of the channel.

In order to calculate the above system, we consider the computational domain with size of $L \times H$, and the periodic boundary condition with a constant pressure difference $\Delta p$ is applied at the inlet and outlet of the domain [49] (see Fig. 15). Also, the periodic condition is used for the temperature at the inlet and the outlet of the domain. In addition, the bounce-back scheme [33] and the iso-thermal condition [21] are used at the bottom and top walls. At $t=0$, the fluid velocity and the temperature are set to $\boldsymbol{u}=\mathbf{0}$ and $T=T_{\mathrm{s}}=0$, respectively, and the particle is stationary. We define the reference Reynolds number 
$R e_{\text {ref }}$ as follows:

$$
R e_{\text {ref }}=\frac{U_{\text {ref } H}}{\nu},
$$

where $U_{\text {ref }}$ is the cross-sectional-averaged flow speed corresponding to the Poiseuille flow induced by the pressure gradient $-\Delta p / L$ as follows:

$$
U_{\text {ref }}=\frac{1}{12 \rho_{\mathrm{f}} \nu} \frac{\Delta p}{L} H^{2} .
$$

In this study, we set $U_{\text {ref }}=7.13 \times 10^{-2} \mathrm{~m} / \mathrm{s}$. Therefore, the governing parameters of the system are $\operatorname{Re} e_{\text {ref }}=208$ and $\operatorname{Pr}=\nu / \alpha=19.2$.

In the present simulation, we slightly modify the LBM in order to make the fluid velocity $\boldsymbol{u}$ periodic at the inlet and the outlet of the domain, while in the original LBM the fluid momentum $\rho \boldsymbol{u}$ is periodic. The modifications are shown in Appendix B. In addition, the Lagrangian points approximation [19] is used for calculating the internal mass effect given by Eqs. (6) and (9). We set $H=$ $L=280 \Delta x, \Delta p=2.337 \times 10^{-4}, U_{\mathrm{ref}}=0.06365, \tau_{f}=0.7570$, and $\tau_{g}=0.5134$. We change the diameter of the particle in the range of $0.071 \leq D_{\mathrm{s}} / H \leq 0.35$, and we investigate the effects of the diameter ratio $D R=D_{\mathrm{s}} / H$ on the motion of the particle and on the temperature field.

\subsection{Results and discussions}

At first, we show the trajectories of the particle with various diameter ratios in Fig. 16. The initial position of the center of the particle is set to $\left(X_{\mathrm{c}}, Y_{\mathrm{c}}\right)=$ $(0.5 H, 0.4 H)$. We can see from Fig. 16(a) that the particle migrates to each equilibrium position for various diameter ratios, which is between the center line and the bottom wall of the channel, i.e., the Segré-Silberberg effect [50] can be observed. It should be noted that the equilibrium positions are independent of the initial position. From Fig. 16(b), it can be seen that the equilibrium position of the bottom of the particle gets closer to the bottom wall of the channel as $D R$ increases.

Next, we calculate the Nusselt numbers on the bottom and top walls of the channel defined as follows:

$$
N u_{\mathrm{b}}=-\left.\frac{H}{T_{\mathrm{w}}-T_{\mathrm{s}}} \frac{\partial T}{\partial y}\right|_{x=0.5 H, y=0}, \quad N u_{\mathrm{t}}=\left.\frac{H}{T_{\mathrm{w}}-T_{\mathrm{s}}} \frac{\partial T}{\partial y}\right|_{x=0.5 H, y=H},
$$




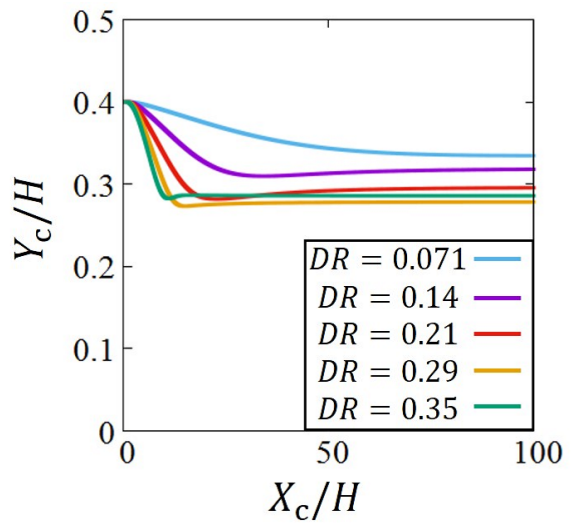

(a)

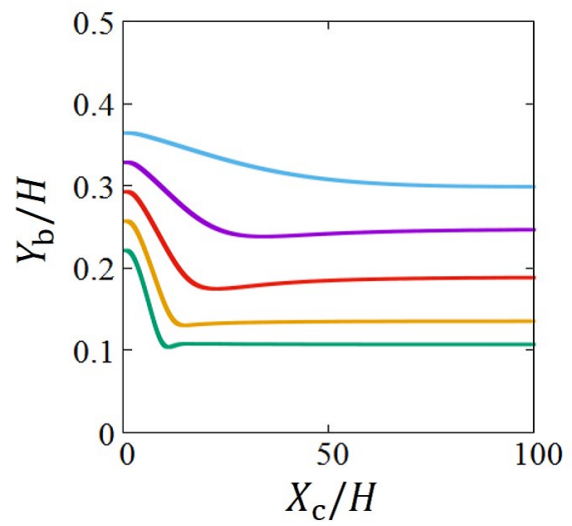

(b)

Figure 16: Trajectories of (a) the center of the particle and (b) the bottom of the particle.

where the derivatives in the above equations are calculated by the first-order

one-sided finite difference approximation of the temperature. Fig. 17 shows the time variations of the Nusselt numbers on the top and bottom walls of the channel. In this figure, the results when the particle is adiabatic are also shown for comparison. We can see from Fig. 17(a) that $N u_{\mathrm{b}}$ tends to each equilibrium value for various diameter ratios, and the equilibrium value increases with $D R$. This means that the endothermic energy amount increases with $D R$. On the other hand, we can see that $N u_{\mathrm{t}}$ decreases monotonically with time, and the differences between the results for various $D R$ are small. This is because the distance between the bottom wall and the particle becomes smaller as $D R$ increases (Fig. 16b), and consequently the bottom wall is easily cooled by the cold particle, while the top wall is too far from the particle even for a large $D R$. In the case where the particle is adiabatic, it can be seen from Fig. 17(b) that both $N u_{\mathrm{b}}$ and $N u_{\mathrm{t}}$ decrease monotonically with time for any of the diameter ratios, although slight differences can be observed between the results of $N u_{\mathrm{b}}$ for various $D R$. It can be considered reasonable that adiabatic particles hardly affect the endothermic energy amount on the walls.

Table 4 shows the equilibrium values of the position of the center of the 

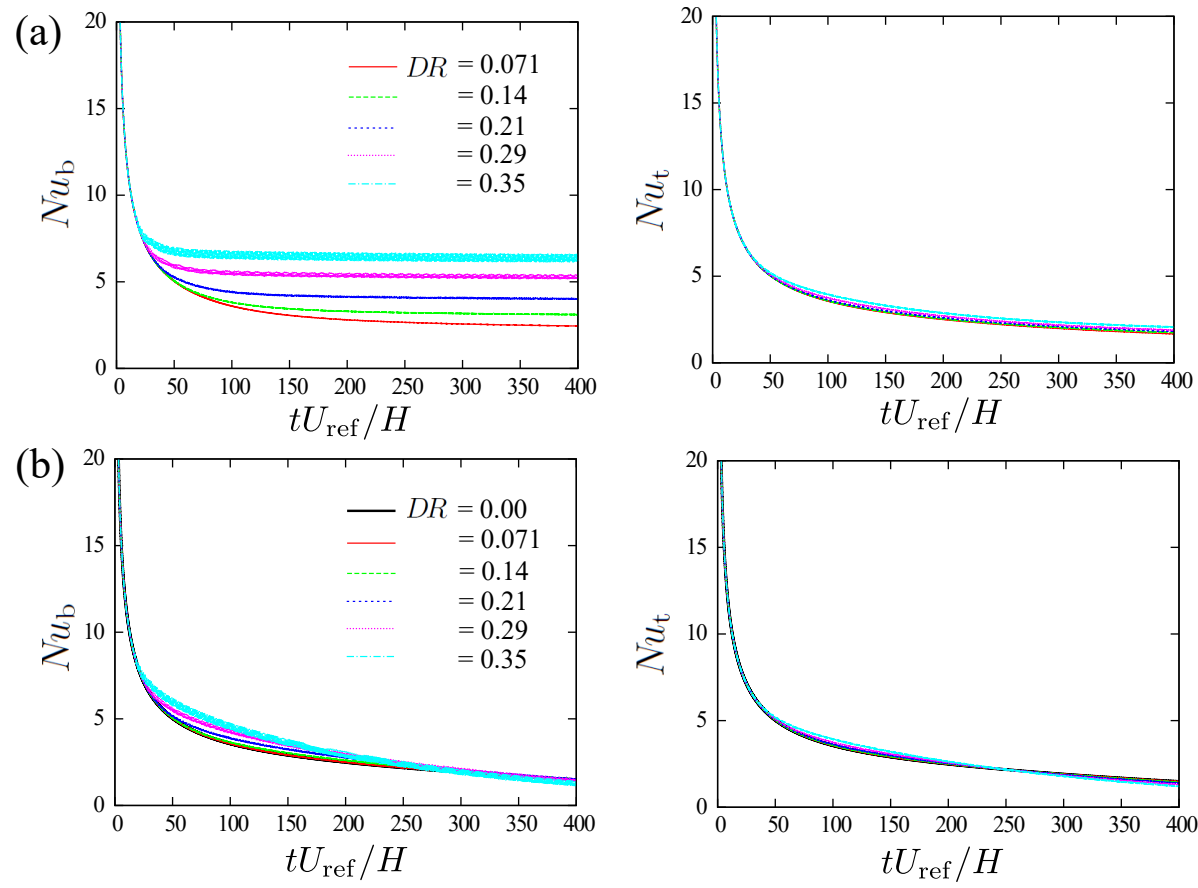

Figure 17: Time variations of the Nusselt numbers of the bottom and top walls of the channel for (a) the cold particle at a constant temperature $T_{\mathrm{s}}=0$ and (b) the adiabatic particle. In (b), $D R=0$ means the case where the channel contains no particle.

particle $\tilde{Y}_{\mathrm{c}}$, the position of the bottom of the particle $\tilde{Y}_{\mathrm{b}}$, the Nusselt number of the bottom wall $\tilde{N} u_{\mathrm{b}}$, and the effective Reynolds number defined by $R e_{\mathrm{eff}}=$ $\tilde{U} H / \nu$, where $\tilde{U}$ is the cross-sectional-averaged flow speed for the particle-fluid mixture at the inlet in the steady state. It should be noted that since $N u_{\mathrm{b}}$ oscillates periodically depending on the horizontal position of the particle, the mean value of the maximum and the minimum is shown in this table. We can see from Table 4 that $\tilde{Y}_{\mathrm{b}}$ decreases and $\tilde{N} u_{\mathrm{b}}$ increases with $D R$, as shown in Figs. 16(b) and 17(a), too. In addition, we can see that $R e_{\text {eff }}$ decreases with $D R$. This is because $\tilde{U}$ becomes smaller than $U_{\text {ref }}$ due to the disturbance by the particle, since the pressure difference $\Delta p$ is constant. This means that if the flow rate of the channel is fixed, the pressure drop should increase with $D R$.

Finally, we show snapshots of the temperature fields from the initial state 
Table 4: The equilibrium values of the position of the center of the particle $\tilde{Y}_{\mathrm{c}}$, the position of the bottom of the particle $\tilde{Y}_{\mathrm{b}}$, the Nusselt number of the bottom wall $\tilde{N} u_{\mathrm{b}}$, and the effective Reynolds number $R e_{\mathrm{eff}}$.

\begin{tabular}{ccccc}
\hline$D R$ & $\tilde{Y}_{\mathrm{c}}$ & $\tilde{Y}_{\mathrm{b}}$ & $\tilde{N} u_{\mathrm{b}}$ & $R e_{\mathrm{eff}}$ \\
\hline 0.071 & 0.335 & 0.299 & 2.41 & 207 \\
0.14 & 0.319 & 0.247 & 3.09 & 205 \\
0.21 & 0.296 & 0.189 & 3.99 & 198 \\
0.29 & 0.278 & 0.136 & 5.30 & 184 \\
0.35 & 0.286 & 0.107 & 6.19 & 169 \\
\hline
\end{tabular}

to the steady state in Fig. 18. In this figure, the results when the particle is adiabatic are also shown for comparison. We can see from Fig. 18(a) that the fluid in the channel is gradually heated, while the fluid around the particle is kept cold. In addition, the gradation of the temperature from the wall to the particle is wavy due to the disturbance by the particle. Comparing Figs. 18(a) and (b), we find that the wavy pattern in the gradation of the temperature is very similar at an early stage $\left(t^{*}=80\right)$. This wavy pattern should be a cause of the difference between the results when the particle is adiabatic as shown in Fig. 17(b). In Fig. 18(b), however, the high-temperature area diffuses more rapidly and widely than that in Fig. 18(a). This result suggests an advantage of ice slurry flow that it can transport cold thermal energy for much longer period and distance.

\section{Conclusions}

We constructed a thermal immersed boundary-lattice Boltzmann method for moving-boundary flows with the Dirichlet and Neumann conditions. The present method incorporates a simple thermal LBM proposed by Inamuro et 730 al. [20] and Yoshino and Inamuro [21] with two types of thermal IBMs, i.e., the multi-direct heat source scheme [9] and the heat flux correction scheme [10] for 
(a)
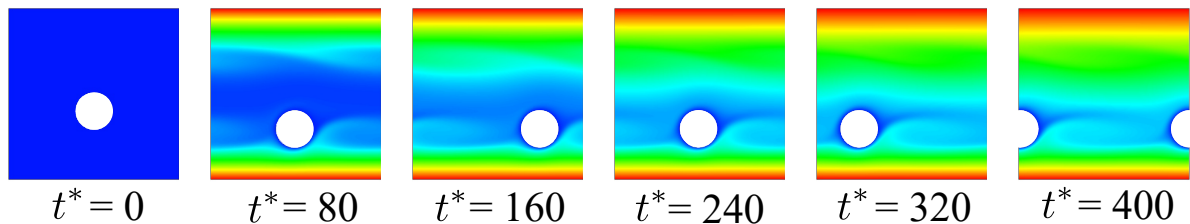

(b)
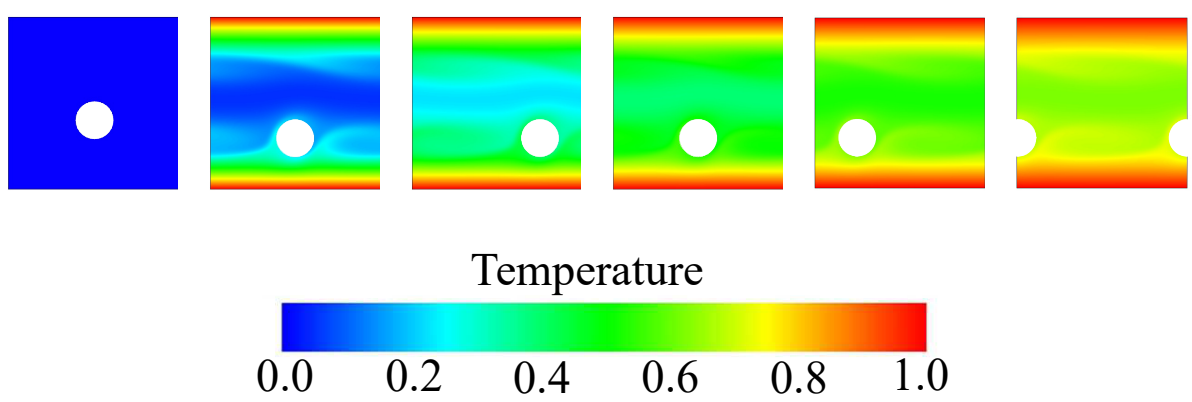

Figure 18: Snapshots of the temperature fields for (a) the cold particle at a constant temperature $T_{\mathrm{s}}=0$ and (b) the adiabatic particle with diameter ratio $D R=0.21$ at various nondimensional time $t^{*}=t U_{\text {ref }} / H$.

calculating the temperature field with the Dirichlet and Neumann conditions, respectively.

We validated the present method through many benchmark problems including stationary and moving boundaries with iso-thermal and iso-heat-flux conditions. As a result, we found that the present method has first-order accuracy for the temperature and the rate of total heat transferred from the boundary to the surrounding fluid, and the present results have good agreement with other numerical results. Also, we investigated the internal heat effect through simulations of moving-boundary flows with heat transfer by using the present method. It was found that there is no need to care about the internal heat effect for the iso-thermal condition with a constant temperature independently of Reynolds and Péclet numbers. However, it was suggested that the internal heat effect should be considered for the iso-thermal condition with a space- and/or time-dependent temperature. On the other hand, in the case for the iso-heatflux condition, it was found that the internal heat effect is significant for Péclet numbers over 10 independently of the Reynolds number. In addition, we found 
that Lagrangian points approximation gives an unphysical oscillation with very high frequency in the calculation of the internal heat effect at a high Péclet number. The remedy of the serious problem remains in future work.

We applied the present method to an interesting example of a movingboundary flow with heat transfer, i.e., a two-dimensional thermal flow in a heated channel with moving cold particles, which is a simplified model of ice slurry flow. As a result, we found that the Nusselt number on the channel wall increases as the ratio of the diameter of the particle to the channel width increases, since a larger particle gets closer to the wall due to the Segré-Silberberg effect [50]. In addition, we found that ice slurry flow can transport cold thermal energy for much longer period and distance than particle-fluid mixture with adiabatic particles. For further investigations, we would like to calculate the channel flows with two or more particles in every length $L$ of the channel, to extend the system in three-dimension, and to consider the effects of melting/solidification of the particle and natural convection.

\section{Acknowledgement}

The authors would like to thank Prof. H. Kumano at Aoyama Gakuin Uni765 versity and Prof. T. Asaoka at Shinshu University for valuable comments to this work.

\section{Appendix A. Nondimensional variables}

In Section 3, we use the following nondimensional variables defined by a characteristic length $\hat{H}_{0}$, a characteristic particle speed $\hat{c}$, a characteristic time scale $\hat{t}_{0}=\hat{H}_{0} / \hat{U}_{0}$ where $\hat{U}_{0}$ is a characteristic flow speed, a reference fluid density $\hat{\rho}_{\mathrm{f} 0}$, a characteristic temperature difference $\Delta \hat{T}_{0}$, a reference temperature $\hat{T}_{0}$, and 
a reference specific heat at constant pressure $\hat{c}_{\text {pfo }}$ :

$$
\begin{array}{lll}
\boldsymbol{c}_{i}=\hat{\boldsymbol{c}}_{i} / \hat{c}, & \boldsymbol{x}=\hat{\boldsymbol{x}} / \hat{H}_{0}, & t=\hat{t} / \hat{t}_{0}, \\
\begin{array}{l}
\Delta x=\Delta \hat{x} / \hat{H}_{0}, \\
f_{i}=\hat{f}_{i} / \hat{\rho}_{\mathrm{f} 0},
\end{array} & g_{i}=\left(\hat{g}_{i}-\hat{T}_{0}\right) / \Delta \hat{T}_{0}, & \\
\rho=\hat{\rho} / \hat{\rho}_{\mathrm{f} 0}, & p=\hat{p} /\left(\hat{\rho}_{\mathrm{f} 0} \hat{c}^{2}\right), & \boldsymbol{u}=\hat{\boldsymbol{u}} / \hat{c}, \\
T=\left(\hat{T}-\hat{T}_{0}\right) / \Delta \hat{T}_{0}, & \boldsymbol{h}=\hat{\boldsymbol{h}} /\left(\hat{\rho}_{\mathrm{f} 0} \hat{c}_{\mathrm{pf} 0} \Delta \hat{T}_{0} \hat{c}\right), & \\
\rho_{\mathrm{f}}=\hat{\rho}_{\mathrm{f}} / \hat{\rho}_{\mathrm{f} 0}, & \nu=\hat{\nu} /\left(\hat{c} \hat{H}_{0}\right), & \\
\lambda_{\mathrm{f}}=\hat{\lambda}_{\mathrm{f}} /\left(\hat{\rho}_{\mathrm{f} 0} \hat{c}_{\mathrm{pf} 0} \hat{c} \hat{H}_{0}\right), & c_{\mathrm{pf}}=\hat{c}_{\mathrm{pf}} / \hat{c}_{\mathrm{pf} 0}, & \\
\boldsymbol{g}=\hat{\boldsymbol{g}} \hat{H}_{0} /\left(\hat{\rho}_{\mathrm{f} 0} \hat{c}^{2}\right), & q=\hat{q} \hat{H}_{0} /\left(\hat{\rho}_{\mathrm{f} 0} \hat{c}_{\mathrm{pf} 0} \Delta \hat{T}_{0} \hat{c}\right), & \\
\boldsymbol{X}_{k}=\hat{\boldsymbol{X}}_{k} / \hat{H}_{0}, & \boldsymbol{U}_{k}=\hat{\boldsymbol{U}}_{k} / \hat{c}, & \\
T_{k}^{\mathrm{d}}=\left(\hat{T}_{k}^{\mathrm{d}}-\hat{T}_{0}\right) / \Delta \hat{T}_{0}, & H_{k}^{\mathrm{d}}=\hat{H}_{k}^{\mathrm{d}} /\left(\hat{\rho}_{\mathrm{f} 0} \hat{c}_{\mathrm{pf} 0} \Delta \hat{T}_{0} \hat{c}\right) . &
\end{array}
$$

Note that the circumflex represents 'dimensional.' It should be noted that the time step $\Delta \hat{t}$ is equal to the time span during which the particles travel one lattice spacing, that is, $\Delta \hat{x} / \Delta \hat{t}=\hat{c}$. We can easily obtain $\Delta t=S h \Delta x$ (where $\left.S h=\hat{H}_{0} /\left(\hat{t}_{0} \hat{c}\right)=\hat{U}_{0} / \hat{c}\right)$ from the above relation.

In Sections 4 and 5, we use the following nondimensional variables:

$$
\begin{array}{lll}
D_{\mathrm{s}}=\hat{D}_{\mathrm{s}} / \hat{H}_{0}, & U_{\infty}=\hat{U}_{\infty} / \hat{c}, & \\
T_{\infty}=\left(\hat{T}_{\infty}-\hat{T}_{0}\right) / \Delta \hat{T}_{0}, & T_{\mathrm{s}}=\left(\hat{T}_{\mathrm{s}}-\hat{T}_{0}\right) / \Delta \hat{T}_{0}, & \\
R_{1}=\hat{R}_{1} / \hat{H}_{0}, & R_{2}=\hat{R}_{2} / \hat{H}_{0}, & U_{1}=\hat{U}_{1} / \hat{c} \\
T_{1}=\left(\hat{T}_{1}-\hat{T}_{0}\right) / \Delta \hat{T}_{0}, & T_{2}=\left(\hat{T}_{2}-\hat{T}_{0}\right) / \Delta \hat{T}_{0}, & \\
\alpha_{\mathrm{g}}=\hat{\alpha}_{\mathrm{g}} \hat{H}_{0} / \hat{c}^{2}, & \beta=\hat{\beta} \Delta \hat{T}_{0}, & T_{\mathrm{f}}=\left(\hat{T}_{\mathrm{f}}-\hat{T}_{0}\right) / \Delta \hat{T}_{0}, \\
h^{\mathrm{d}}=\hat{h}^{\mathrm{d}} /\left(\hat{\rho}_{\mathrm{f} 0} \hat{c}_{\mathrm{pf} 0} \Delta \hat{T}_{0} \hat{c}\right), & \Delta T=\Delta \hat{T} / \Delta \hat{T}_{0}, & \\
A=\hat{A} / \hat{H}_{0}, & f=\hat{f} \hat{t}_{0}, & \\
H=\hat{H} / \hat{H}_{0}, & T_{\mathrm{w}}=\left(\hat{T}_{\mathrm{w}}-\hat{T}_{0}\right) / \Delta \hat{T}_{0}, & \Delta p=\Delta \hat{p} /\left(\hat{\rho}_{\mathrm{f} 0} \hat{c}^{2}\right), \\
L=\hat{L} / \hat{H}_{0} . &
\end{array}
$$




\section{Appendix B. Modifications of the LBM in Section 5}

In Section 5, instead of using Eq. (15), we use the equilibrium distribution function of the incompressible model [51] as follows:

$$
f_{i}^{\mathrm{eq}}(p, \boldsymbol{u})=E_{i}\left[3 p+3 \boldsymbol{c}_{i} \cdot \boldsymbol{u}+\frac{9}{2}\left(\boldsymbol{c}_{i} \cdot \boldsymbol{u}\right)^{2}-\frac{3}{2} \boldsymbol{u} \cdot \boldsymbol{u}\right] .
$$

According to the above modification, Eqs. (19) and (27) are modified as follows:

$$
\begin{aligned}
& \boldsymbol{u}=\sum_{i=1}^{9} f_{i} \boldsymbol{c}_{i}, \\
& f_{i}(\boldsymbol{x}, t+\Delta t)=f_{i}^{*}(\boldsymbol{x}, t+\Delta t)+3 \Delta x E_{i} \boldsymbol{c}_{i} \cdot \boldsymbol{g}(\boldsymbol{x}, t+\Delta t) .
\end{aligned}
$$

\section{References}

[1] C. S. Peskin, Flow patterns around heart valves: A numerical method, J. Comput. Phys. 10 (1972) 252-271.

[2] C. S. Peskin, Numerical analysis of blood flow in the heart, J. Comput. Phys. 25 (1977) 220-252.

[3] R. Mittal, G. Iaccarino, Immersed boundary methods, Annu. Rev. Fluid Mech. 37 (2005) 239-261.

[4] J. Kim, H. Choi, An immersed-boundary finite-volume method for simulation of heat transfer in complex geometries, Korean Soc. Mech. Eng. Int. J. 18 (2004) 1026-1035.

[5] J. R. Pacheco, A. Pacheco-Vega, T. Rodić, R. E. Peck, Numerical simulations of heat transfer and fluid flow problems using an immersed-boundary finite-volume method on nonstaggered grids, Numer. Heat Transfer B 48 (2005) 1-24.

[6] D. Pan, An immersed boundary method on unstructured Cartesian meshes for incompressible flows with heat transfer, Numer. Heat Transfer B 49 (2006) 277-297. 
[7] N. Zhang, Z. C. Zheng, S. Eckels, Study of heat-transfer on the surface of a circular cylinder in flow using an immersed-boundary method, Int. J. Heat Fluid Flow 29 (2008) 1558-1566.

[8] Z. G. Feng, E. E. Michaelides, Heat transfer in particulate flows with Direct Numerical Simulation (DNS), Int. J. Heat Mass Transfer 52 (2009) 777-786.

[9] Z. L. Wang, J. R. Fan, K. Luo, K. Cen, Immersed boundary method for the simulation of flows with heat transfer, Int. J. Heat Mass Transfer 52 (2009) 4510-4518.

[10] W. W. Ren, C. Shu, W. M. Yang, An efficient immersed boundary method for thermal flow problems with heat flux boundary conditions, Int. J. Heat Mass Transfer 64 (2013) 694-705.

[11] S. Chen, G. D. Doolen, Lattice Boltzmann method for fluid flows, Annu. Rev. Fluid Mech. 30 (1998) 329-364.

[12] H. K. Jeong, H. S. Yoon, M. Y. Ha, M. Tsutahara, An immersed boundarythermal lattice Boltzmann method using an equilibrium internal energy density approach for the simulation of flows with heat transfer, J. Comput. Phys. 229 (2010) 2526-2543.

[13] S. K. Kang, Y. A. Hassan, A direct-forcing immersed boundary method for the thermal lattice Boltzmann method, Comput. Fluids 49 (2011) 36-45.

[14] H. Zhang, A. Yu, W. Zhong, Y. Tan, A combined TLBM-IBM-DEM scheme for simulating isothermal particulate flow in fluid, Int. J. Heat Mass Transfer 91 (2015) 178-189.

[15] A. Eshghinejadfard, D. Thévenin, Numerical simulation of heat transfer in particulate flows using a thermal immersed boundary lattice Boltzmann method, Int. J. Heat Fluid Flow 60 (2016) 31-46.

[16] J. Wu, Y. Cheng, L. A. Miller, An iterative source correction based immersed boundary-lattice Boltzmann method for thermal flow simulations, Int. J. Heat Mass Transfer 115 (2017) 450-460. 
[17] Y. Hu, D. C. Li, S. Shu, X. D. Niu, An efficient immersed boundary-lattice Boltzmann method for the simulation of thermal flow problems, Commun. Comput. Phys. 20 (2016) 1210-1257.

[18] Y. Wang, C. Shu, L. M. Yang, Boundary condition-enforced immersed boundary-lattice Boltzmann flux solver for thermal flows with Neumann boundary conditions, J. Comput. Phys. 306 (2016) 237-252.

[19] K. Suzuki, T. Inamuro, Effect of internal mass in the simulation of a moving body by the immersed boundary method, Comput. Fluids 49 (2011) 173187.

[20] T. Inamuro, M. Yoshino, H. Inoue, R. Mizuno, F. Ogino, A lattice Boltzmann method for a binary miscible fluid mixture and its application to a heat-transfer problem, J. Comput. Phys. 179 (2002) 201-215.

830 [21] M. Yoshino, T. Inamuro, Lattice Boltzmann simulations for flow and heat/mass transfer problems in a three-dimensional porous structure, Int. J. Numer. Methods Fluids 43 (2003) 183-198.

[22] Z. L. Wang, J. R. Fan, K. Luo, Combined multi-direct forcing and immersed boundary method for simulating flows with moving particles, Int. J. Multiphase Flow 34 (2008) 283-302.

[23] M. Kauffeld, M. J. Wang, V. Goldstein, K. E. Kasza, Ice slurry applications, Int. J. Refr. 33 (2010) 1491-1505.

[24] Y. H. Qian, D. d'Humières, P. Lallemand, Lattice BGK models for NavierStokes equation, Europhys. Lett. 17 (1992) 479-484.

[25] T. Inamuro, Lattice Boltzmann methods for viscous fluid flows and for two-phase fluid flows, Fluid Dyn. Res. 38 (2006) 641-659.

[26] M. Junk, A. Klar, L.-S. Luo, Asymptotic analysis of the lattice Boltzmann equation, J. Comput. Phys. 210 (2005) 676-704. 
[27] T. Inamuro, M. Yoshino, F. Ogino, Accuracy of the lattice Boltzmann method for small Knudsen number with finite Reynolds number, Phys. Fluids 9 (1997) 3535-3542.

[28] C. S. Peskin, The immersed boundary method, Acta Numerica 11 (2002) $479-517$.

[29] I. Orlanski, A simple boundary condition for unbounded hyperbolic flows, J. Comput. Phys. 21 (1976) 251-269.

[30] S. C. R. Dennis, J. D. Hudson, N. Smith, Steady laminar forced convection from a circular cylinder at low Reynolds numbers, Phys. Fluids 11 (1968) 933-940.

[31] M. W. Chang, B. A. Finlayson, Heat transfer in flow past cylinders at $R e<150$ - Part I. Calculations for constant fluid properties, Numer. Heat Transfer 12 (1987) 179-195.

[32] H. Gan, J. Chang, J. J. Feng, H. H. Hu, Direct numerical simulation of the sedimentation of solid particles with thermal convection, J. Fluid Mech. 481 (2003) 385-411.

[33] S. Succi, The Lattice Boltzmann Equation for Fluid Dynamics and Beyond, (Oxford: Oxford University Press), 2001.

[34] A. ten Cate, C. H. Nieuwstad, J. J. Derksen, H. E. A. Van den Akker, Particle imaging velocimetry experiments and lattice-Boltzmann simulations on a single sphere settling under gravity, Phys. Fluids 14 (2002) 4012-4025.

865 [35] Z. G. Feng, E. E. Michaelides, Robust treatment of no-slip boundary condition and velocity updating for the lattice-Boltzmann simulation of particulate flows, Comput. Fluids 38 (2009) 370-381.

[36] T. Krüger, F. Varnik, D. Raabe, Efficient and accurate simulations of deformable particles immersed in a fluid using a combined immersed boundary 

3485-3505.

[37] K. Suzuki, T. Inamuro, A higher-order immersed boundary-lattice Boltzmann method using a smooth velocity field near boundaries, Comput. Fluids 76 (2013) 105-115.

[38] K. Suzuki, I. Okada, M. Yoshino, Accuracy of the laminar boundary layer on a flat plate in an immersed boundary-lattice Boltzmann simulation, J. Fluid Sci. Technol. 11 (2016) 16-00488 (17pp).

[39] M.-C. Lai, C. S. Peskin, An immersed boundary method with formal second-order accuracy and reduced numerical viscosity, J. Comput. Phys. 160 (2000) 705-719.

[40] R. D. Guy, D. A. Hartenstine, On the accuracy of direct forcing immersed boundary methods with projection methods, J. Comput. Phys. 229 (2010) 2479-2496.

[41] Z. S. Yu, X. M. Shao, A. Wachs, A fictitious domain method for particulate flows with heat transfer, J. Comput. Phys. 217 (2006) 424-452.

[42] A. Wachs, Rising of 3D catalyst particles in a natural convection dominated flow by a parallel DNS method, Comput. Chem. Eng. 35 (2011) 2169-2185.

[43] S. Haeri, J. S. Shrimpton, A new implicit fictitious domain method for the simulation of flow in complex geometries with heat transfer, J. Comput. Phys. 237 (2013) 21-45.

[44] R. P. Bharti, R. P. Chhabra, V. Eswaran, A numerical study of the steady forced convection heat transfer from an unconfined circular cylinder, Heat Mass Transfer 43 (2007) 639-648.

[45] J. S. Yoo, Dual free-convective flows in a horizontal annulus with a constant heat flux wall, Int. J. Heat Mass Transfer 46 (2003) 2499-2503. 
[46] Y. Peng, C. Shu, Y. T. Chew, Simplified thermal lattice Boltzmann model for incompressible thermal flows, Phys. Rev. E 68 (2003) 026701 (8pp).

[47] A. C. S. Monteiro, P. K. Bansal, Pressure drop characteristics and rheological modeling of ice slurry flow in pipes, Int. J. Refr. 33 (2010) 1523-1532.

[48] H. Kumano, T. Hirata, R. Shouji, M. Shirakawa, Experimental study on heat transfer characteristics of ice slurry, Int. J. Refr. 33 (2010) 1540-1549.

[49] T. Inamuro, K. Maeba, F. Ogino, Flow between parallel walls containing the lines of neutrally buoyant circular cylinders, Int. J. Multiphase Flow 26 (2000) 1981-2004.

[50] G. Segré, A. Silberberg, Radial particle displacements in Poiseuille flow of suspensions, Nature 189 (1961) 209-210.

[51] X. Y. He, L. S. Luo, Lattice Boltzmann model for the incompressible Navier-Stokes equation, J. Stat. Phys. 88 (1997) 927-944. 